\title{
PLK1 stabilizes a MYC-dependent kinase network in aggressive B cell lymphomas
}

\author{
Yuan Ren, ${ }^{1}$ Chengfeng Bi, ${ }^{2}$ Xiaohong Zhao, ${ }^{1}$ Tint Lwin, ${ }^{1}$ Cheng Wang, ${ }^{2}$ Ji Yuan, ${ }^{2}$ Ariosto S. Silva, ${ }^{3}$ Bijal D. Shah, ${ }^{4}$ Bin Fang, ${ }^{5}$ \\ Tao Li, ${ }^{1}$ John M. Koomen, ${ }^{5}$ Huijuan Jiang, ${ }^{1,6}$ Julio C. Chavez, ${ }^{4}$ Lan V. Pham, ${ }^{7}$ Praneeth R. Sudalagunta, ${ }^{3}$ Lixin Wan, ${ }^{8}$ \\ Xuefeng Wang, ${ }^{9}$ William S. Dalton, ${ }^{4}$ Lynn C. Moscinski, ${ }^{1}$ Kenneth H. Shain, ${ }^{4}$ Julie Vose, ${ }^{10}$ John L. Cleveland, ${ }^{11}$ \\ Eduardo M. Sotomayor, ${ }^{12}$ Kai Fu, ${ }^{2}$ and Jianguo Tao ${ }^{1,4}$
}

\begin{abstract}
'Department of Laboratory Medicine and Hematopathology, Moffitt Cancer Center \& Research Institute, Tampa, Florida, USA. ${ }^{2}$ Department of Pathology and Microbiology, University of Nebraska Medical Center, Omaha, Nebraska, USA. ${ }^{3}$ Department of Cancer Imaging and Metabolism, ${ }^{4}$ Department of Malignant Hematology, and ${ }^{5}$ Proteomics Core Facility, Moffitt Cancer Center \& Research Institute, Tampa, Florida, USA. ${ }^{6}$ Tianjin Medical School, Tianjin, China. ${ }^{7}$ Department of Hematopathology, The University of Texas MD Anderson Cancer Center, Houston, Texas, USA. ${ }^{8}$ Department of Molecular Oncology and ${ }^{9}$ Department of Biostatics and Bioinformatics, Moffitt Cancer Center \& Research Institute, Tampa, Florida, USA. ${ }^{10}$ Department of Internal Medicine, University of Nebraska Medical Center, Omaha, Nebraska, USA. "Department of Tumor Biology, Moffitt Cancer Center \& Research Institute, Tampa, Florida, USA. ${ }^{2}$ Department of Hematology \& Oncology, George Washington University, Washington, DC, USA
\end{abstract}

\begin{abstract}
Concordant activation of MYC and BCL-2 oncoproteins in double-hit lymphoma (DHL) results in aggressive disease that is refractory to treatment. By integrating activity-based proteomic profiling and drug screens, polo-like kinase-1 (PLK1) was identified as an essential regulator of the MYC-dependent kinome in DHL. Notably, PLK1 was expressed at high levels in DHL, correlated with MYC expression, and connoted poor outcome. Further, PLK1 signaling augmented MYC protein stability, and in turn, MYC directly induced PLK1 transcription, establishing a feed-forward MYC-PLK1 circuit in DHL. Finally, inhibition of PLK1 triggered degradation of MYC and of the antiapoptotic protein MCL-1, and PLK1 inhibitors showed synergy with BCL-2 antagonists in blocking DHL cell growth, survival, and tumorigenicity, supporting clinical targeting of PLK1 in DHL.
\end{abstract}

\section{Introduction}

MYC is a transcription factor that promotes oncogenesis by activating and repressing target genes that control cell growth, metabolism, and division $(1,2)$. MYC is deregulated in a large proportion of aggressive B cell lymphomas. Although MYC chromosomal translocations are the defining feature of Burkitt lymphoma (BL), MYC is also deregulated in a large proportion of aggressive $\mathrm{B}$ cell lymphomas (3), in which MYC is associated with an aggressive course of disease, chemoresistance, and poor prognosis (4). Despite current modes of intensive chemotherapy, targeted B cell therapy (e.g., rituximab), and radiation, overall survival (OS) in $\mathrm{B}$ cell lymphoma patients with high MYC activity is dismal, and it is still unclear which direct MYC-induced transcription targets promote aggressive disease.

Double-hit lymphoma (DHL) is a subgroup of aggressive B cell lymphoma originally defined as having both MYC and BCL2 chromosomal translocations, which have a rapidly progressing clinical course, are refractory to aggressive treatment, and have short survival $(5,6)$. Over time, the definition of DHL was expanded to include diffuse large B cell lymphoma (DLBCL) having MYC trans-

\section{Related Commentary: p. 5206}

Authorship note: YR, CB, and XZ contributed equally to this work. Conflict of interest: The authors have declared that no conflict of interest exists. License: Copyright 2018, American Society for Clinical Investigation.

Submitted: June 1, 2018; Accepted: September 20, 2018

Reference information: / Clin Invest. 2018;128(12):5517-5530.

https://doi.org/10.1172/JCl122533. location combined with translocations involving either $B C L 2$ or $B C L 6$ as well as DLBCL that cooverexpress MYC and BCL-2 oncoproteins via other means (double-protein-expression lymphomas [DELs]) $(6,7)$. Overall, approximately $20 \%-30 \%$ of DLBCLs overexpress both MYC and BCL-2 or have MYC and BCL2 gene rearrangements, and with standard therapy for non-Hodgkin lymphoma (e.g., R-CHOP), both DHL patient types have a worse prognosis than patients without these alterations, with median OS of only 5 to 24 months $(8,9)$. Given that both DHL and DEL share a rapidly progressing clinical course, are refractory to treatment, and are currently considered incurable, we included both of these germinal center-originated large B cell lymphomas subtypes $(6,7,10-15)$ in our analyses and have designated both types as DHL in this study.

Chromosomal translocation, gene amplification, mutations in signaling pathways, and alterations in protein stability all promote MYC overexpression in tumors $(1,16)$. Notably, the addiction of MYC-driven tumors to this oncoprotein, including MYC-driven lymphomas (17), has made MYC an appealing target for cancer therapy. However, as a transcription factor, MYC is widely considered "undruggable" (18). Identifying critical molecules and signaling processes required for MYC action in DHL provides an alternative strategy for targeting MYC-driven lymphoma. However, the antiapoptotic functions of BCL-2 add a substantial layer of complexity to the pathobiology and therapy of DHL. Like other prosurvival proteins, such as MCL-1 and BCL-XL, BCL-2 functions by binding to $\mathrm{BH} 3$ domain-only proapoptotic factors that counteract their activity (19). Accordingly, BCL-2-targeting strategies have focused on small molecules that disrupt these proteinprotein interactions to restore the apoptotic response in cancer 
A

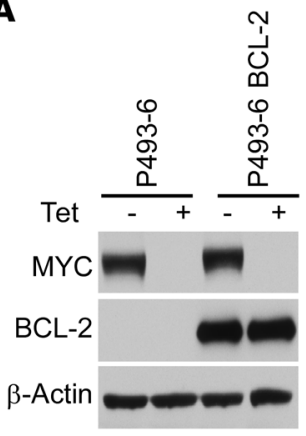

D

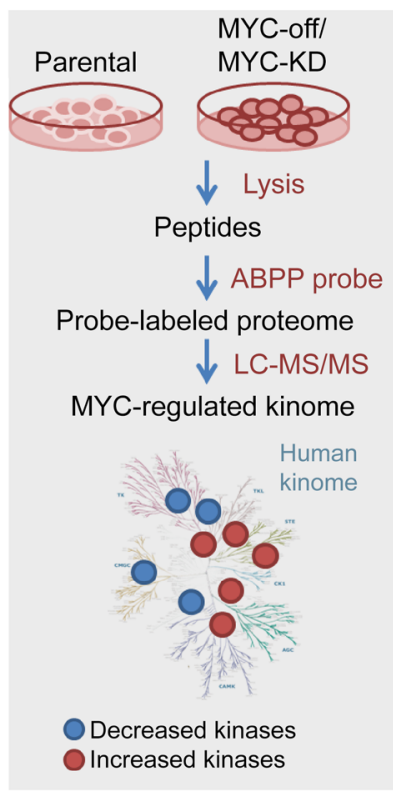

B

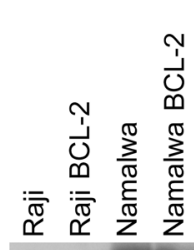

C
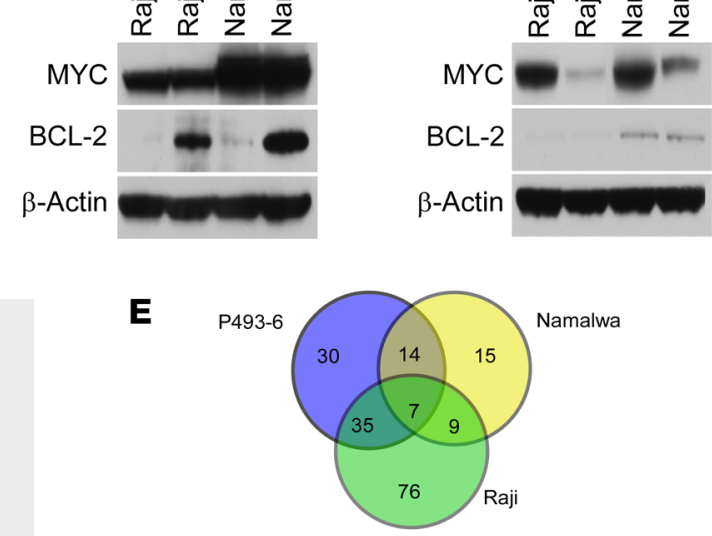

MYC up-regulated kinases

\begin{tabular}{|c|c|c|ccc|}
\hline 7 & 9 & 14 & \multicolumn{3}{|c|}{35} \\
\hline ERN1 & M3K2 & KCC4 & CLK3 & BMP2K & LATS1 \\
RN5AK & ACK1 & CDK7 & ATR & OXSR1 & M4K5 \\
S6A4RI & PRP4B & TOPK & E2AK4 & KS6A5 & SRPK2 \\
PK2 & CDK13 & CHK2 & MARK1 & TLK1 & TAOK1 \\
CHK1 & VRK1 & MAST4 & NEK4 & OBSCN & EPHA3 \\
AURKA & KS6A1 & NEK2 & ABL1 & PRKDC & PAK2 \\
M3K5 & NEK3 & KKCC1 & WEE1 & CD11B & M3K4 \\
& CDK16 & AURKC & STRAA & CSK21 & \\
& PK3CG & PLK1 & MAST2 & NUAK2 \\
& & AURKB & KKCC2 & EF2K \\
& & TYRO3 & SRPK1 & STK16 \\
& & FGR & KPCI & MHK3 \\
& & PRKX & PKN2 & E2AK3 \\
& & M3K11 & MET & TLK2 \\
\hline
\end{tabular}

Figure 1. MYC-regulated kinome in lymphoma.

(A) Generation of isogenic BCL-2-expressing (MYC Tet-repressible) P493-6 B lymphoma cells. (B) Generation of isogenic BCL-2-expressing Raji and Namalwa BL cells. (C) Generation of isogenic CRISPR/cas9-mediated MYC KO/KD derivatives of Raji and Namalwa BL cells. (D) Schematic work flow of ABPP studies. (E) Overlap of kinases whose activity is upregulated by MYC in P493-6, Namalwa, and Raji BL cells (upper panel) and list of MYC-upregulated kinases (lower panel). (F) KEGG pathway analysis of MYC-upregulated kinome in models of DHL. See complete unedited blots in the supplemental material.

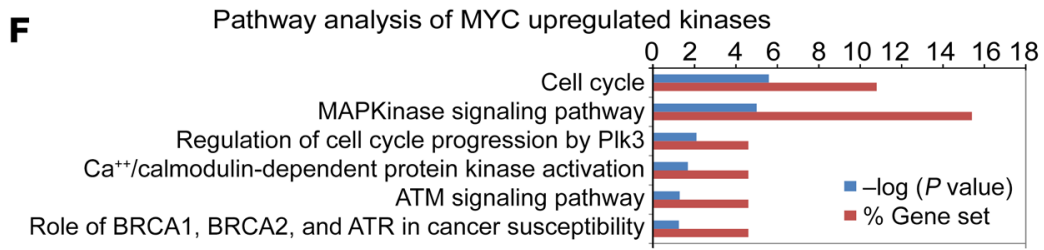

cells (20). BCL-2 inhibitors, such as venetoclax (ABT-199), have recently been approved for the treatment of chronic lymphocytic leukemia (CLL) and are currently being tested in clinical trials for other hematological malignances (21). This suggests that if effective therapies could be found to disable MYC, their combination with BCL-2 inhibitors might be efficacious in the treatment of DHL.

Protein kinases play key regulatory roles in a number of biological processes (22), and deregulation of protein kinase signaling is a hallmark of cancer. Accordingly, kinases have proven to be highly promising clinical targets (23). However, the contribution of kinases to DHL and their potential as therapeutic targets is largely unknown. Using chemical proteomics and unbiased protein kinase inhibitor drug screens on a platform that recapitulates the bone marrow tumor microenvironment (24), as well as a series of isogenic and inducible MYC/BCL-2 lymphoma lines, DHL cell lines, and primary DHL patient-derived xenografts (PDX), we defined signaling kinase pathways altered in DHL. These analyses identified a major kinase network involving polo-like kinase-1
(PLK1)as a hub for the MYC-dependent kinome in DHL. Importantly, analyses of the regulation and role of PLK1 revealed a feed-forward MYC-PLK1 circuit in DHL and showed that PLK1 is a therapeutic vulnerability for DHL, particularly in combination with BCL-2 antagonists.

\section{Results}

The MYC-driven kinome in B cell lymphomas. To identify the MYC-dependent kinome in B cell lymphoma, we capitalized on P493-6 B lymphoma cells that bear a doxycycline-repressed MYC transgene (25) and engineered these cells to also overexpress BCL-2 to generate isogenic MYC on/off and BCL-2 high/low B lymphoma cell lines (Figure 1A). As BLs have high MYC levels and express low levels of BCL-2, we also engineered $2 \mathrm{BL}$ cell lines, Raji and Namalwa, to overexpress BCL-2 (Figure 1B). Finally, we applied CRISPR/cas9 editing to knockdown (KD) MYC expression in Raji and Namalwa BL (Figure 1C). Using these isogenic cells, we then performed activity-based protein profiling (ABPP) to identify 
A

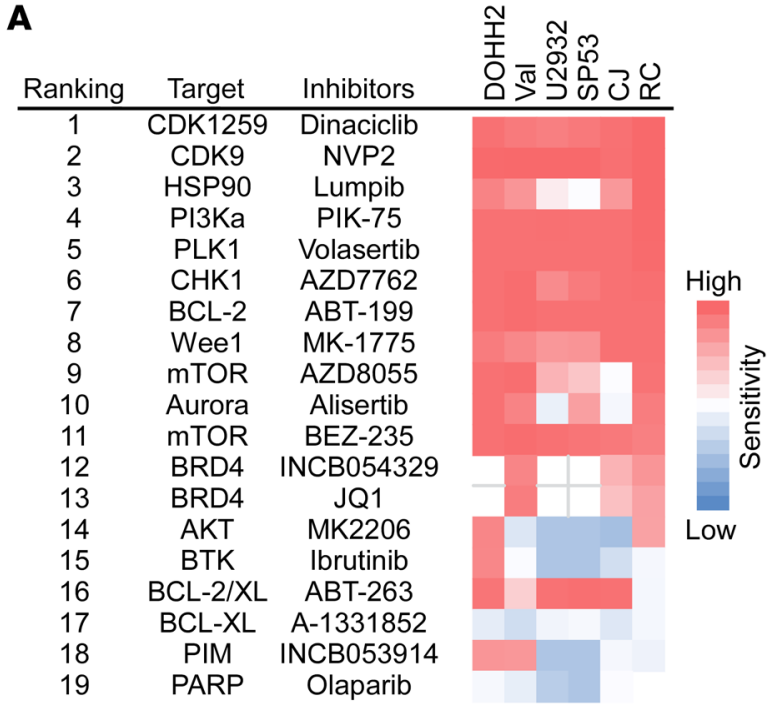

B

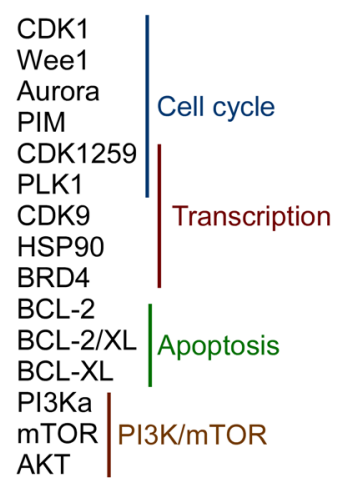

D

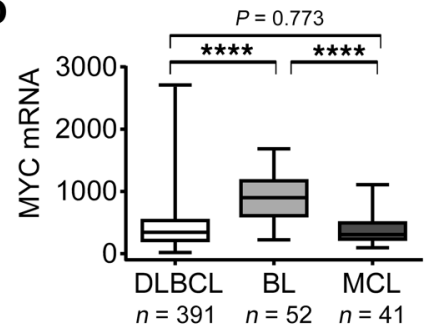

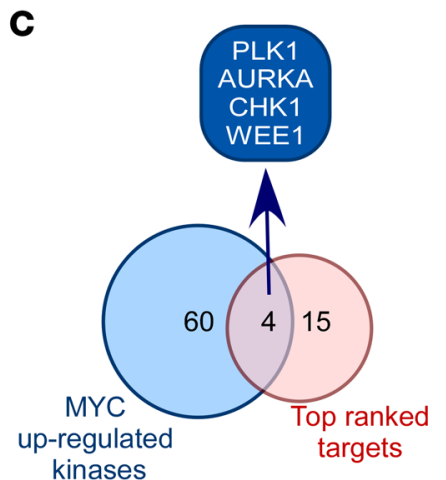

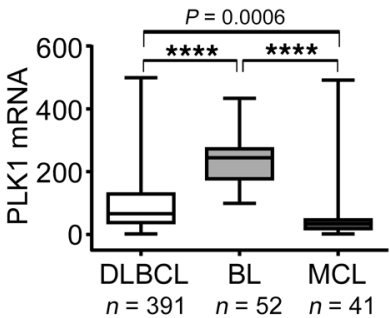

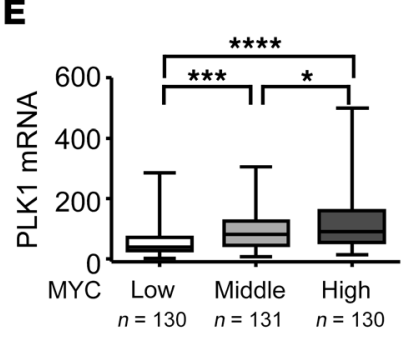

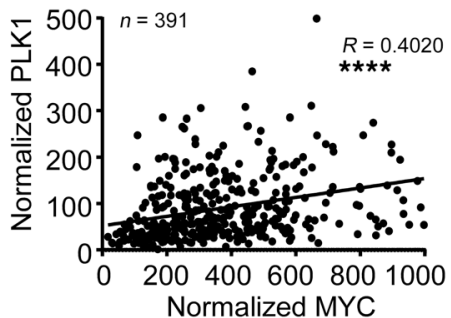

$\mathbf{F}$

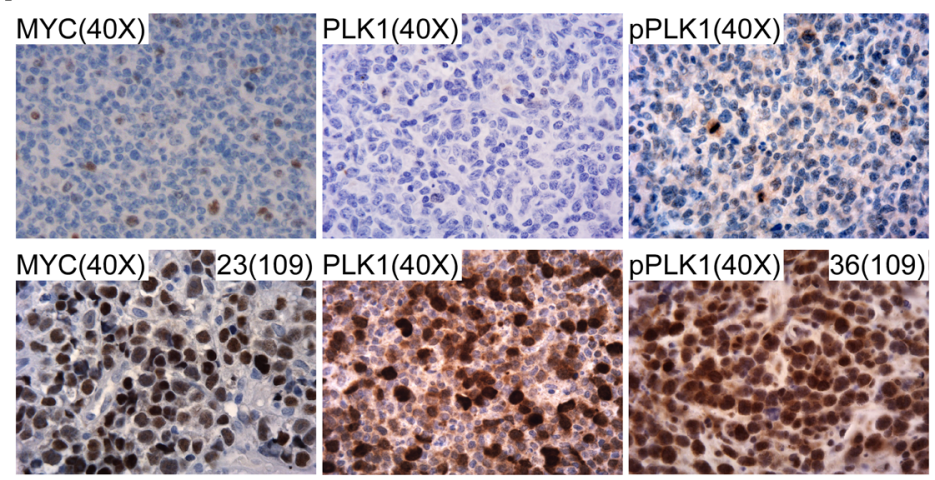

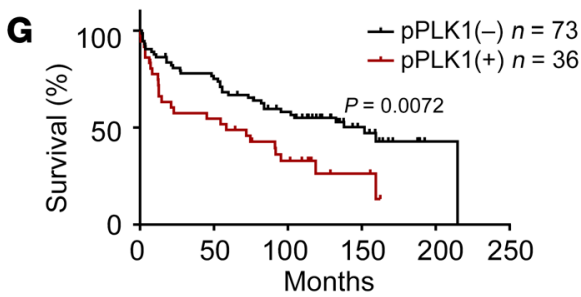
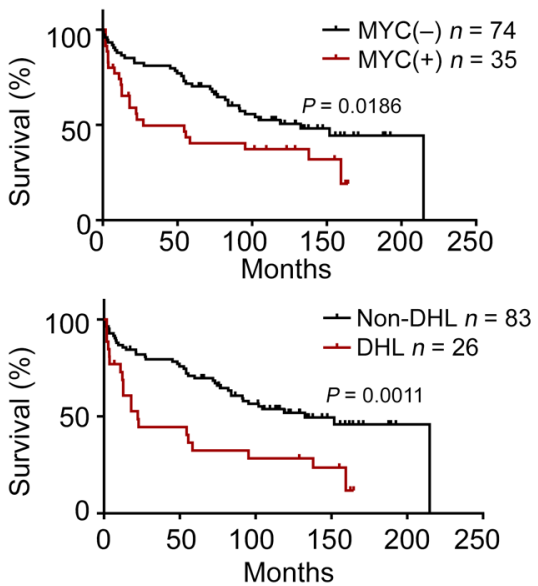

Figure 2. PLK1 is elevated in DHL, connotes poor survival, and is a therapeutic vulnerability for DHL cells. (A) Functional drug screens in DHL cells (DOHH2, Val, U2932, SP53, CJ, and RC); summary of top 19 ranked small molecules and corresponding targets, as represented in an IC ${ }_{50}$ heatmap format. (B) Top ranked small-molecule inhibitors of DHL are categorized according to their target signaling pathways. (C) Overlap of MYC-upregulated kinases by ABPP (see Figure 1E) and top ranked small-molecule inhibitors having activity versus DHL. (D) MYC and PLK1 mRNA levels were analyzed in a gene expression profiling data set of DLBCL, BL, and mantle cell lymphoma (MCL). ${ }^{* * *} P<0.0001$. (E) Correlation of the mRNA levels of MYC and $P L K 1$ in DLBCL. ${ }^{*} P=0.0406$; ${ }^{* *} P=0.0005$; ${ }^{* * * * P}<0.0001$. (F) Representative images of MYC, PLK1, and p-PLK1 IHC staining in reactive lymphoma nodes (top panels) versus DHL (bottom panels). Original magnification, $\times 40$. (C) Clinical outcome of 109 cases of DLBCL patients treated with R-CHOP when correlated with $p$-PLK1 and MYC expression and DHL classification. Comparisons among group means in $\mathbf{D}$ and $\mathbf{E}$ were performed by 1-way ANOVA, followed by Tukey's test for multiple-comparison test.

MYC-regulated kinases. To this end, a desthiobiotin-ATP probe that selectively binds to the active sites of ATP-binding proteins was used, followed by identification and quantification using liquid chromatography-tandem mass spectrometry (LC-MS/MS)
(Figure 1D) (24). Using the 3 paired $\mathrm{MYC}^{\text {hi }}$ and $\mathrm{MYC}^{\text {lo }} \mathrm{B}$ lymphoma cell lines, ABPP identified a total of 715 peptides from 263 protein kinases. Assessing the relative kinase activities of $\mathrm{MYC}^{\text {hi }}$ versus $\mathrm{MYC}^{\mathrm{lo}}$ lymphoma cells revealed that a select set of kinas- 

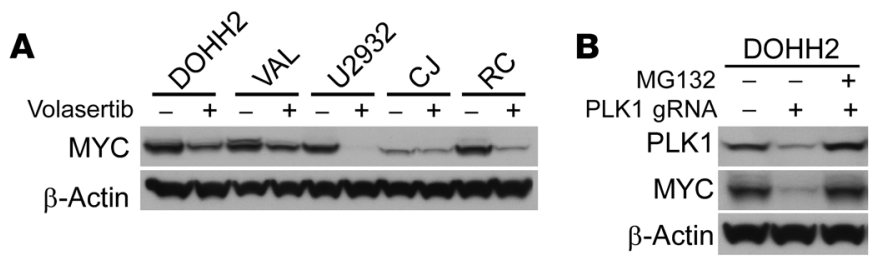

D
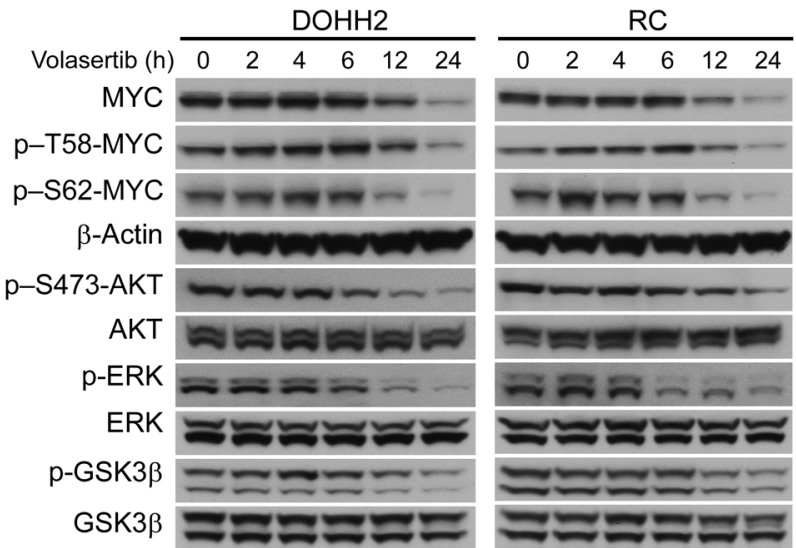

$\mathbf{F}$
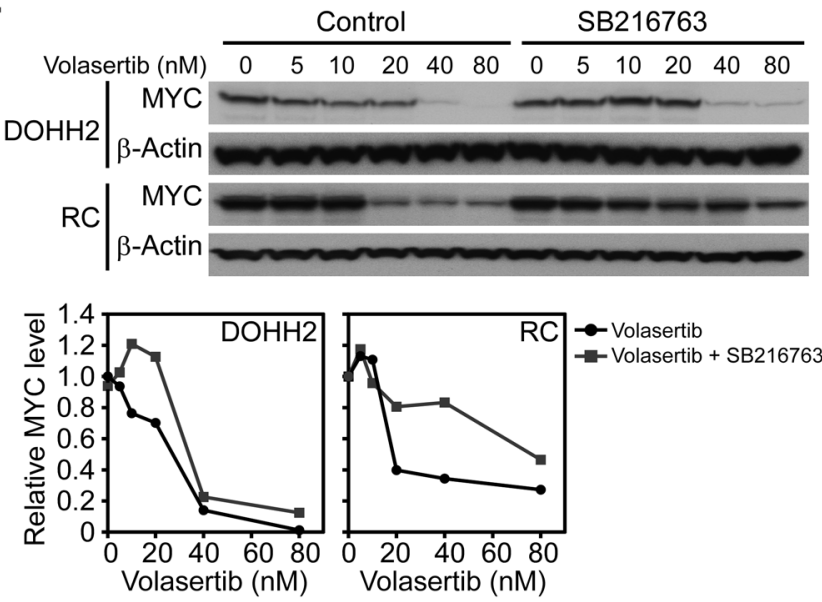
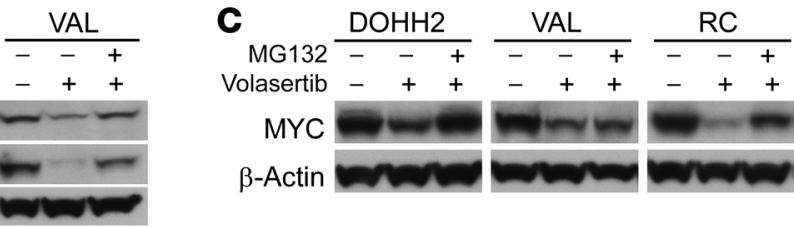

E

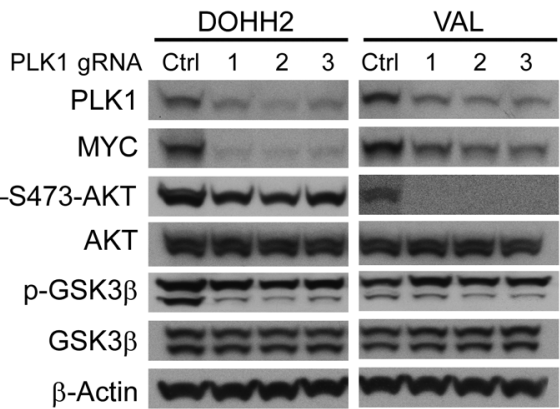

G

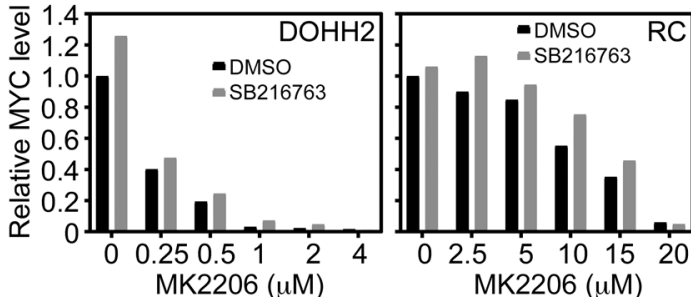

DMSO

SB216763

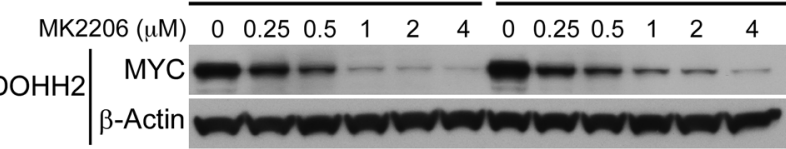

$\begin{array}{lllllllllllll}\text { MK2206 }(u M) & 0 & 2.5 & 5 & 10 & 15 & 20 & 0 & 2.5 & 5 & 10 & 15 & 20\end{array}$

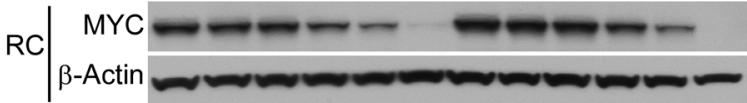

Figure 3. PLK1 sustains MYC protein stability by activating an AKT-GSK3 $\beta$ circuit. (A) Volasertib treatment (20 nM, 24 hours) reduces steady-state levels of MYC protein in DOHH2, VAL, U2932, CJ, and RC DHL cells. (B) PLK1 KO by CRISPR/cas9 editing provokes marked reductions in MYC protein levels that can be restored by treatment (6 hours) with the proteasome inhibitor MC132 (10 $\mu \mathrm{M})$. (C) Reductions in MYC protein provoked by volasertib treatment (20 $\mathrm{nM}, 24$ hours) are at least partially blocked by pretreatment (6 hours) with MC132 (10 $\mu$ M). (D) PLK1 inhibition (volasertib, $20 \mathrm{nM}$ ) triggers reductions in MYC and blocks AKT, GSK3 $\beta$, and ERK1/2 activation in DHL cells. Cells were treated for the indicated intervals and assessed for levels of MYC, $p-T 58-M Y C$, p-S62-MYC, $\beta$-actin, p-S473-AKT, total AKT, p-ERK1/2, total ERK1/2, p-GSK3 $\beta$, and total GSK3 $\beta$. (E) PLK1 KO triggers reductions in MYC and in activation of AKT and GSK3 $\beta$ in DOHH2 and VAL DHL cells. (F and G) GSK3 $\beta$ inhibition with SB216763 (5 $\mu$ M) (F) or AKT inhibition with MK2206 (G) impairs volasertibinduced reductions of MYC protein in DOHH2 and RC DHL cells. (A-G). Data shown are representative of at least 3 independent experiments. See complete unedited blots in the supplemental material.

es are MYC dependent (Figure 1E). Mapping shared kinases in the 3 lymphoma models using Kyoto Encyclopedia of Genes and Genomes (KEGG) pathway analysis identified replicative stress (PLK1/Aurora A), ATM/ATR DNA damage/repair, cell cycle, and MAPK pathways as central signaling hubs and MYC-driven kinases in these DHL models (Figure 1F). In line with these findings, several of these kinases promote cancer cell survival by protecting cells from MYC oncogene-induced replicative stress and promoting DNA repair (26-28).

PLK1, CHK1, WEE1, and Aurora-A are MYC-regulated kinases required for DHL cell survival. To assess whether components of the MYC-regulated kinome identified in the 3 MYC/BCL-2 lymphoma cell line models represented vulnerabilities for DHL, drug screens comprising pf 60 kinase inhibitors, epigenetic drugs, and chemotherapeutics was performed in 6 DHL or DEL cell lines. This screen identified several kinase inhibitors as having potent activity versus DHL cells and showed that inhibitors targeting kinases that regulate the cell cycle, transcription, and the DNA repair pathway had the most potency against DHL cells (Figure 2, A and B). Integrating these drug screen results with MYCupregulated kinases (Figure 1B) revealed that PLK1, CHK1, WEE1, and Aurora A are both MYC-regulated and functionally important 
A
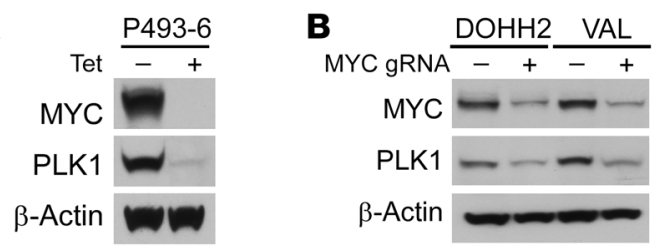

C
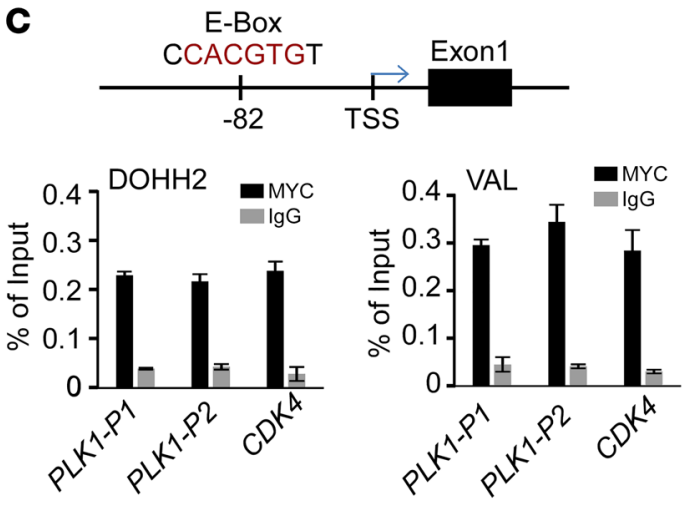

Figure 4. MYC activates PLK1 transcription in DHL. (A) PLK1 protein levels are dependent on MYC. P493-6 cells were cultured with Tet for 72 hours, and a portion were then deprived of Tet for 24 hours. Levels of MYC, PLK1, and $\beta$-actin were determined by Western blot. (B) MYC KD by CRISPR/ cas9 gene editing provokes reductions in PLK1 protein in DHL DOHH2 and VAL cells. (C) Upper panel: an E-box site that conforms to the preferred binding site for MYC (CACGTC, PLK1-P1) is located approximately 80 base pairs upstream of the PLK1 transcription start site (TSS). Lower panel: ChIP assays revealed MYC binds to this region of the PLK1 promoter in DHL cells. Binding of MYC to the $C D K 4$ promoter was assessed as a positive control. $\mathbf{A}$ and $\mathbf{B}$ are representative of $\mathbf{3}$ independent experiments. Data presented in $\mathbf{C}$ show the mean \pm SD of at least 3 independent experiments. See complete unedited blots in the supplemental material.

kinases for DHL cell survival and that the PLK1 inhibitor volasert$i b$ had the most potent activity in DHL cells (Figure 2C). In accord with these findings, PLK1, CHK1, WEE1, and Aurora A have been shown to be vulnerabilities in other malignancies with $M Y C$ or MYCN involvement (26, 27, 29-32).

PLK1 expression and activity correlate with MYC and connote poor outcome in DHL. We therefore queried PLK1 expression in a gene expression profiling data set that comprises 484 primary $\mathrm{B}$ cell lymphoma samples, including DHL. Notably, PLK1 mRNA levels positively correlated with MYC mRNA expression in B cell lymphomas, and this was especially so in MYC-driven lymphomas, such as BL (Figure 2, D and E). To assess whether PLK1 expression correlated with PLK1 activity, immunohistochemical stains were performed using a tissue microarray comprising primary DLBCL patient samples that included DHL specimens. Compared with reactive lymph nodes, PLK1 and phosphorylated-PLK1 (p-PLK1) levels were significantly higher in DHL and correlated with high levels of MYC (Figure 2F). Finally, elevated levels of both MYC and p-PLK1 are associated with a very poor outcome, and all DHLs expressed high levels of p-PLK1 (Figure 2G). Therefore, elevated PLK1 expression and activity are a hallmark of DHL.

PLK1 sustains MYC activity through GSK3 $\beta$-mediated MYC protein stability in DHL. A PLK1-N-MYC circuit has been reported in neuroblastoma, where PLK1 stabilizes MYC protein half- life indirectly, by phosphorylating the $\mathrm{SCF}^{\mathrm{Fbw}}$ ubiquitin ligase, which triggers FBW7 autopolyubiquitination and its destruction by the proteasome, leading to stabilization of N-MYC (26). To test whether a similar circuit might be manifest in DHL, DHL cell lines (VAL, DOHH2, RC, CJ, U2932) were treated with 2 selective PLK1 inhibitors, volasertib and Ro3280, and effects on MYC protein levels were determined by Western blot. Volasertib and Ro3280 treatment led to time- and dose-dependent declines in MYC protein levels (Figure 3A and Supplemental Figure 1A; supplemental material available online with this article; https://doi.org/10.1172/ JCI122533DS1). Further, efficient genetic depletion of PLK1 using CRISPR/cas9 editing in VAL and DOHH2 DHL cells led to similar marked reductions in the steady-state levels of MYC protein (Figure 3, B and E). Thus, PLK1 expression and activity are required to sustain MYC expression in DHL.

The mechanism by which PLK1 sustains MYC expression was not transcriptional, as quantitative real-time PCR (qRT-PCR) analyses revealed that PLK1 inhibition has modest effects on MYC mRNA levels (Supplemental Figure 1B). Consistent with this notion, reductions in MYC protein provoked by either PLK1 KO or PLK1 inhibition (by volasertib) were attenuated by pretreatment (30 minutes) with the proteasome inhibitor MG132 (Figure 3, B and C). Furthermore, analyses of MYC protein turnover in DHL cells (DOHH2, RC, and VAL) treated with volasertib with or without cycloheximide (CHX) revealed that inhibition of PLK1 significantly shortened the half-life of the MYC protein in DOHH2, RC, and VAL cells (Supplemental Figure 1C). Therefore, PLK1 activity controls MYC protein turnover.

MYC protein stabilization is controlled by phosphorylation at threonine 58 (T58) and serine 62 (S62) (16). Time-course analysis showed that treatment of DOHH2, RC, and U2932 cells with volasertib led to increases in p-T58-MYC and p-S62-MYC by 6 hours and to decreased levels at later intervals, which were due to reductions in MYC protein levels (Figure 3D and Supplemental Figure 1D). We also observed decreased phosphorylation of active ERK, AKT, and GSK3 $\beta$ following PLK1 inhibition with volasertib treatment in these DHL cells (Figure 3D and Supplemental Figure 1D). ERK directs phosphorylation of S62-MYC, and this primes MYC for phosphorylation of T58 by GSK3 $\beta$, which targets MYC for ubiquitination and degradation by FBW7 $(16,33)$. Notably, efficient inhibition of ERK (with PD032590) did not affect levels of MYC or of p-S62-MYC in all 3 DHL cell lines tested (Supplemental Figure 1E). Thus, ERK activation does not contribute to the control of MYC turnover in DHL.

AKT, through its regulation of GSK3 $\beta$, has been shown to affect T58-MYC phosphorylation and promote stability of the MYC oncoprotein in various cancers (34-36). Notably, efficient inhibition of AKT by treatment with MK2206 (as judged by levels of p-S473-AKT) reduced levels of active pGSK3 $\beta$ and MYC protein in DHL cells (Supplemental Figure 1F). Further, depletion of PLK1 in DOHH2 and VAL DHL cells using 3 different guide RNAs (gRNAs) led to reductions in MYC, p-AKT, and p-GSK3 $\beta$ in DHL cells (Figure 3E). Finally, inhibition of GSK3 $\beta$ by treatment of DHL cells with SB216763 or LiCl attenuated both volasertib- and AKT inhibitor-induced (MK2206) reductions in MYC protein levels (Figure 3, F and G, and Supplemental Figure 2A). Thus, a PLK1-toAKT-to-GSK3 $\beta$ circuit controls MYC protein levels in DHL. 
A

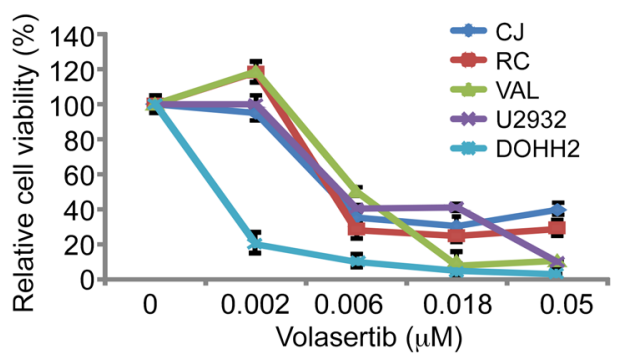

Volasertib (0.12 $\mu \mathrm{M})$
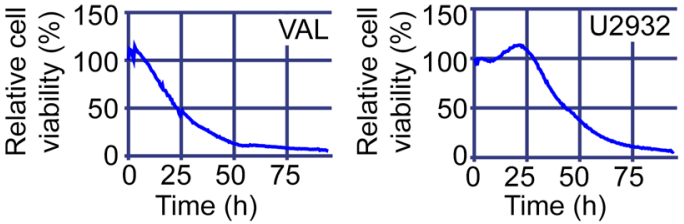

D
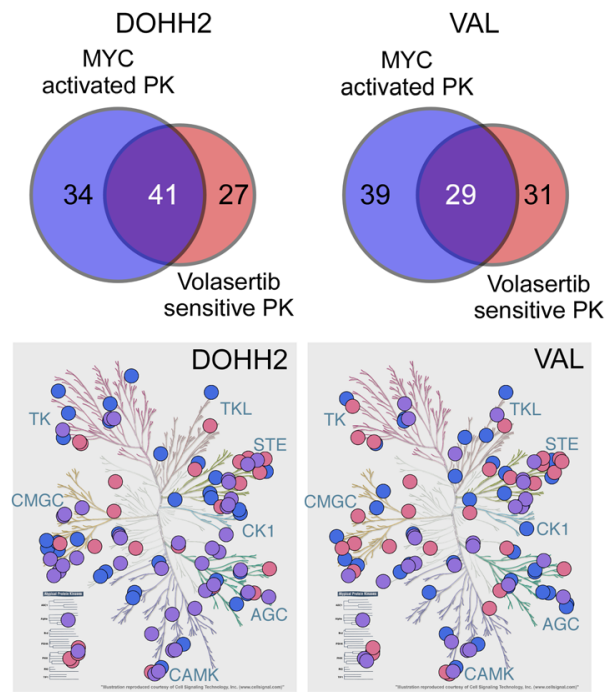

MYC activated PKs

Volasertib sensitive PKs

C Common
B

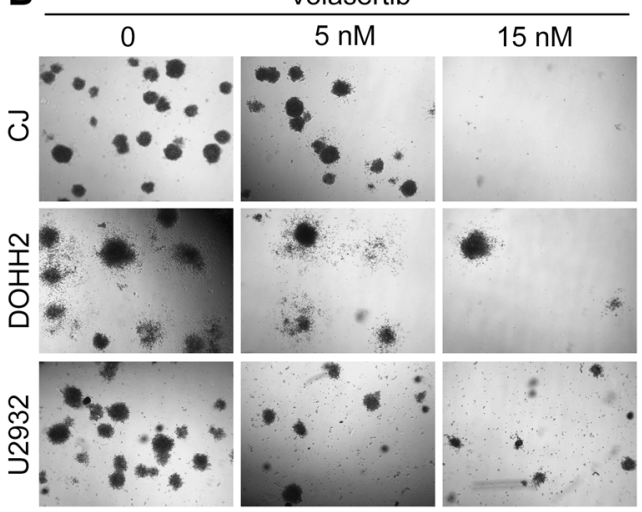

C

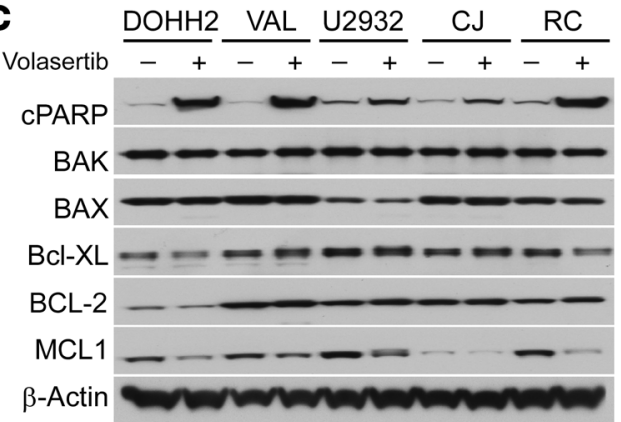

E
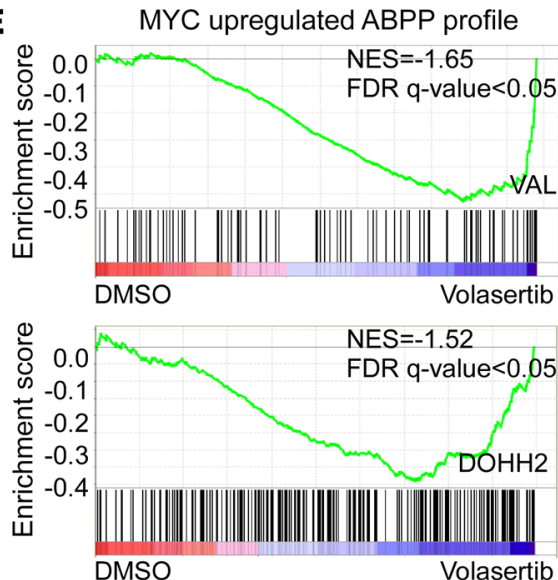

Figure 5. PLK1 function is required for the maintenance of DHL. (A) Volasertib treatment compromises DHL cell survival. Dose response and time course of volasertib treatment on cell viability of DHL and BL cells, as indicated by percentage of cell viability, as determined by cell titer blue assays (upper panel) and imaging-based drug screening assay (lower panels). (B) Volasertib treatment inhibits the clonogenic capacity of DHL cells seeded in methylcellulose. Original magnification, $\times 40$. (C) Volasertib treatment ( $20 \mathrm{nM}$ for 24 hours) provokes the cleavage of PARP and suppresses MCL-1 protein levels in DHL cells. Western blot analysis of the indicated cells was performed to assess the effects of PLK1 inhibition on the expression of BCL-2 family members. (D) Overlap of MYC-upregulated kinases and PLK1-dependent kinases in DOHH2 and VAL DHL cells. MYC-activated protein kinases (PK) were determined by ABPP after CRISPR/cas9-mediated MYC KO/KD in DHL lines DOHH2 and VAL cells (versus parental cells) and PLK1-senstive protein kinases were determined after 2 hours of volasertib treatment $(20 \mathrm{nM})$ in DOHH2 and VAL cells. $\log _{2}$ fold change of more than 1 indicates increased kinase ATP probe binding with relative increased activity, and $\log _{2}$ fold change of -1 or less indicates decreased ATP probe binding with decreased activity relative to parental cells. Data presented are the average of 3 biological replicates performed in duplicate. Kinome tree illustration reproduced courtesy of Cell Signaling Technology (www.cellsignal.com). (E) GSEA of the MYC-activated ABPP profile (upper panel) and of the ABPP profile changes provoked by PLK1 inhibition (lower panel) establish that volasertib treatment represses MYC-activated kinases (from DOHH2 and VAL MYC-KO ABPP profile). Normalized enrichment score (NES) $=-1.65$ (VAL); NES = $-1.52(\mathrm{DOHH} 2)$. Data shown in $\mathbf{B}$ and $\mathbf{C}$ are representative of at least 3 independent experiments. See complete unedited blots in the supplemental material.

In neuroblastoma, PLK1 stabilizes MYC by promoting autoubiquitylation and proteasome degradation of the $\mathrm{E} 3$ ubiquitin ligase FBW7 (26). In accord with these findings, CRISPR/cas9-directed deletion of FBW7 in U2932 DHL cells blocked volasertib-induced reductions in MYC protein levels (Supplemental Figure 2B). Thus, PLK1 sustains MYC expression through FBW7-mediated MYC protein degradation, and PLK1 inhibitors are an attractive general approach to targeting MYC and MYC-associated malignancies. 

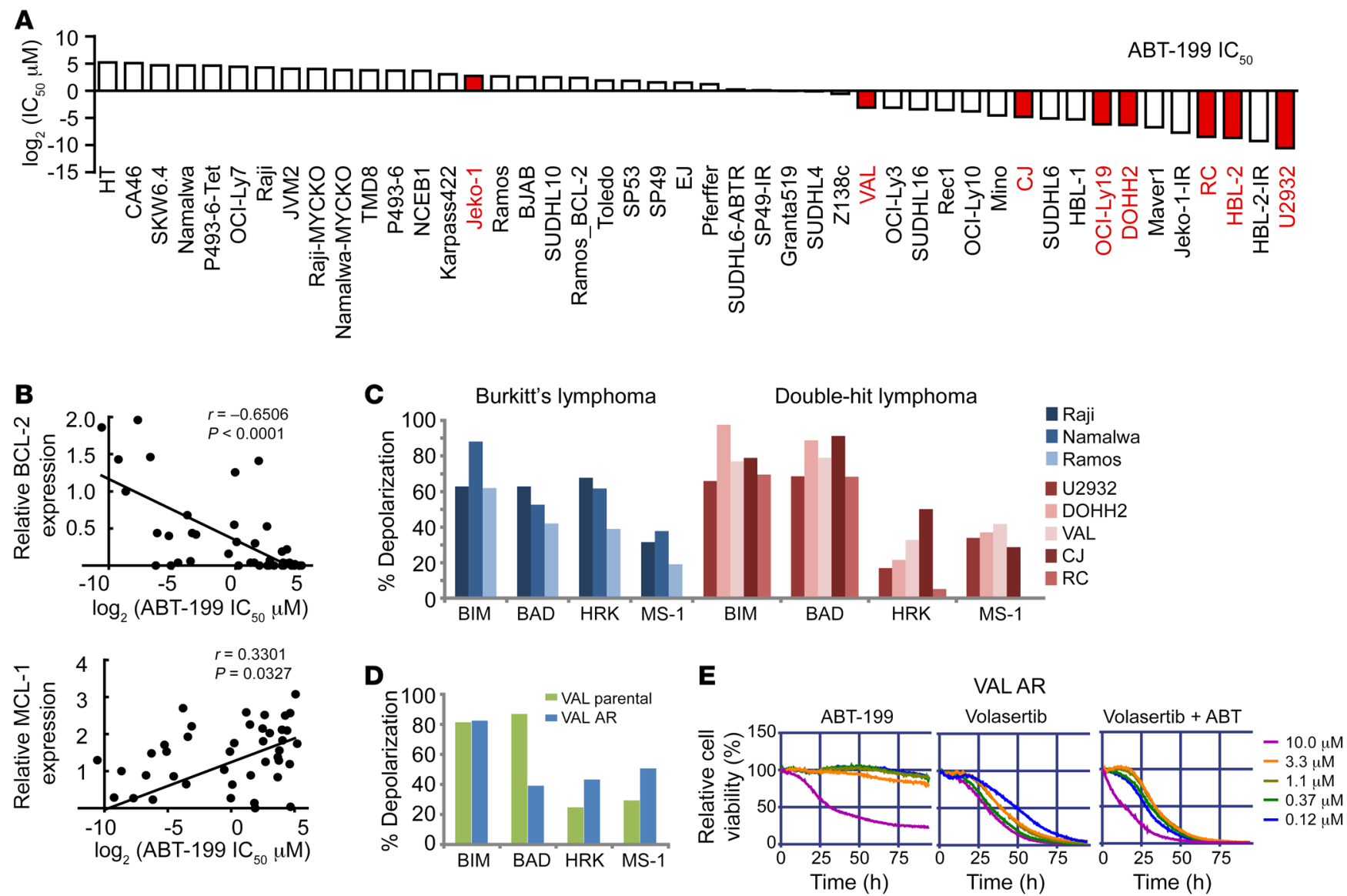

Figure 6. BH3 profiling and sensitivity of DHL and ABT-199-resistant DHL to PLK1 inhibition. (A) $I_{50}$ of the indicated $B$ lymphoma cell lines to ABT199. DHL/DEL cell lines are highlighted in red. (B) Correlation of BCL-2 and MCL-1 protein levels and ABT-199 IC $C_{50}$. IC ${ }_{50}$ values were determined using MTT assays, and protein levels were determined by Western blot. (C) BH3 profiling of DHL (red, MYC ${ }^{\text {hi }} / B C L-2^{\text {hi }}$ ) and BL (blue, MYC ${ }^{\text {hi//BCL- }} 2^{10}$ ) cell lines showing the dependency of most lines to BCL-2 priming. Increased sensitivity (mitochondrial membrane depolarization) to (BAD-HRK) peptides is indicative of BCL-2 dependency; thus, DHL cells are sensitive to ABT-199. (D) BH3 profiling of ABT-199-resistant DHL cells (VAL_AR) reveals a shift to dependency on MCL-1. (E) Viability of VAL_AR cells treated with ABT-199 (left), volasertib (middle), or both volasertib and ABT-199 (right).

MYC activates PLK1 transcription to maintain PLK1 activity in DHL. The positive correlation between MYC and PLK1 in DHL cell lines and primary samples might simply reflect that a higher percentage of these B lymphoma cells are cycling and transiting mitosis. To address whether PLK1 was upregulated in response to MYC, human P493-6 B lymphoma cells were deprived of tetracycline (Tet), which induces the MYC transgene (25). Notably, the induction of MYC protein led to marked increases in the levels of PLK1 protein (Figure 4A). In contrast, suppression of the MYC transgene following readdition of Tet to $\mathrm{P} 493-6 \mathrm{~B}$ cells led to marked decreases in MYC protein and to reductions in PLK1 mRNA and protein and in PLK1 phosphorylation (Supplemental Figure 3, A and B). Further, MYC depletion using CRISPR/cas9 editing resulted in marked reductions in PLK1 levels in DOHH2 and VAL DHL cells and in Raji BL cells (Figure 4B and Supplemental Figure 3C).

Consistent with the notion that MYC activates PLK1 transcription, we identified 2 promoter-regulatory regions in the PLK1 gene that contains 2 canonical MYC E-box-binding sites, including one at -80 base pairs upstream of the PLK1 transcription start site (Supplemental Figure $3 \mathrm{C}$ ). ChIP assays performed in both DOHH2 and VAL DHL cells and in Raji BL cells revealed a significant increase in MYC recruitment to the PLK1 promoter-promixal E-box motif when compared with IgG isotope control (Figure 4C and Supplemental Figure 3D), suggesting that MYC activates PLK1 transcription in MYC-associated lymphomas. Thus, a feed-forward PLK1MYC circuit is manifest in DHL, where PLK1 signaling stabilizes MYC protein, which in turn then drives $P L K 1$ transcription.

$P L K 1$ function is required for DHL cell survival and is a vulnerability for DHL. Treatment of 5 different DHL cell lines with the PLK-1 inhibitor volasertib and cell-viability assays (24) revealed marked time- and dose-dependent sensitivity of DHL, with $\mathrm{IC}_{50}$ ranging from 2 to $6 \mathrm{nM}$ (Figure 5A); thus, PLK1 function is essential for DHL cell survival. Further, clonogenic growth assays revealed that PLK1 inhibition with volasertib dramatically suppressed anchorageindependent cell growth of DHL cells (Figure 5B). Mechanistically, the effects of volasertib on DHL cell survival were linked to apoptosis, with marked cleavage of PARP and significant reductions in MCL-1 (and MYC) protein, but with little or no effect on other apoptosis-related proteins, such as BCL-2, BAX, BAK, and BCL-XL (Figure 5C). Interestingly, volasertib-induced reductions in MCL-1 protein levels in DOHH2 and RC DHL cells were also blocked by pretreatment with the proteasome inhibitor MG132, suggesting 
A

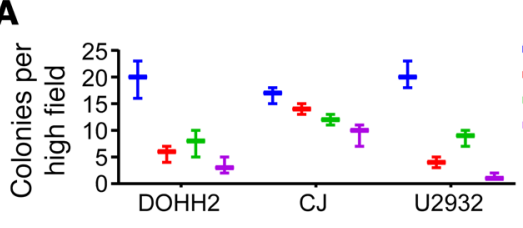

C
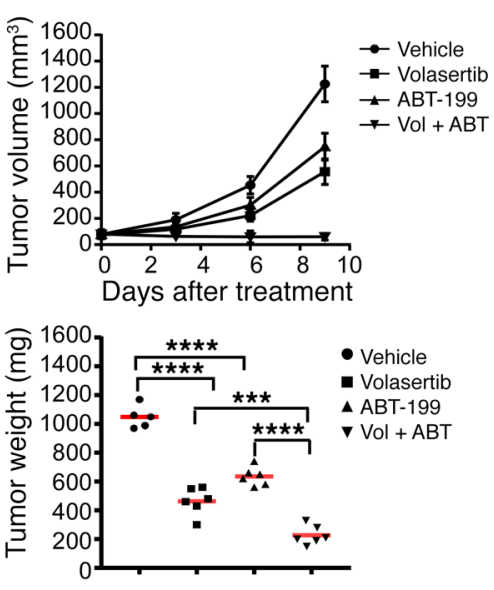

D

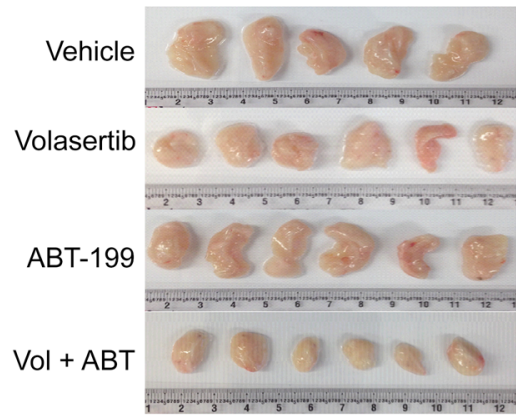

G

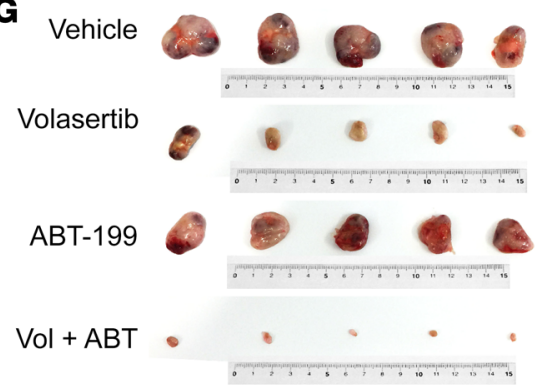

B
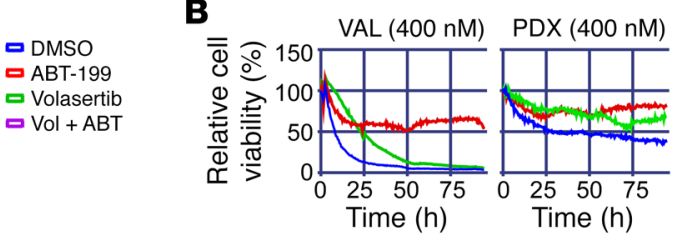

- Volasertib

- ABT-199
- Vol + ABT
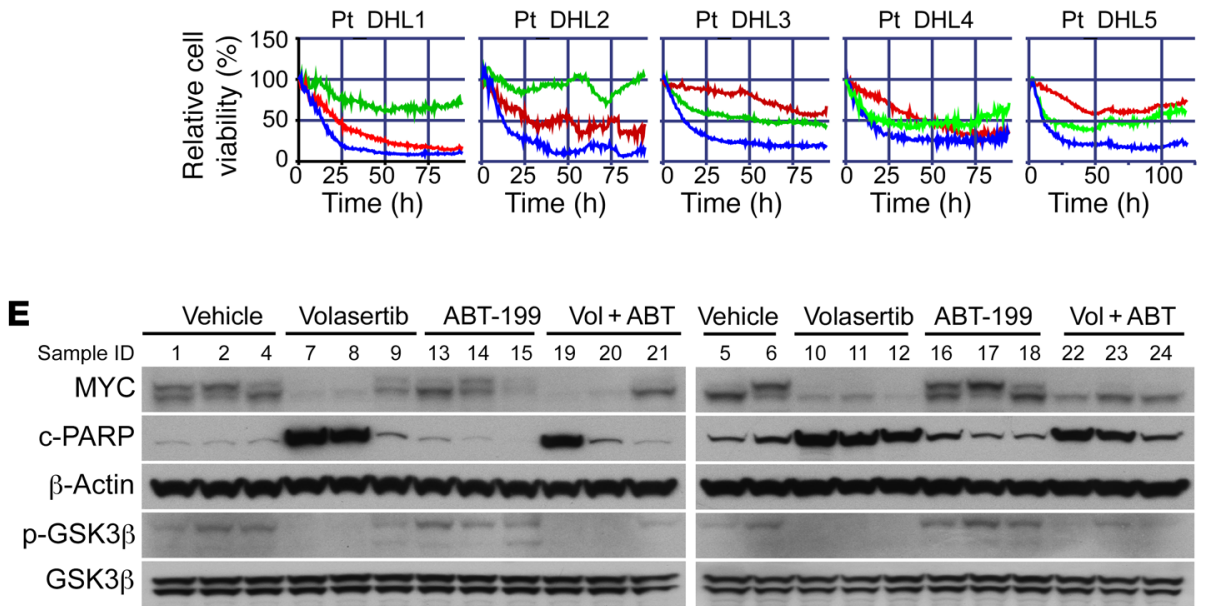

$\mathbf{F}$

H\&E

CD20

MYC

BCL-2
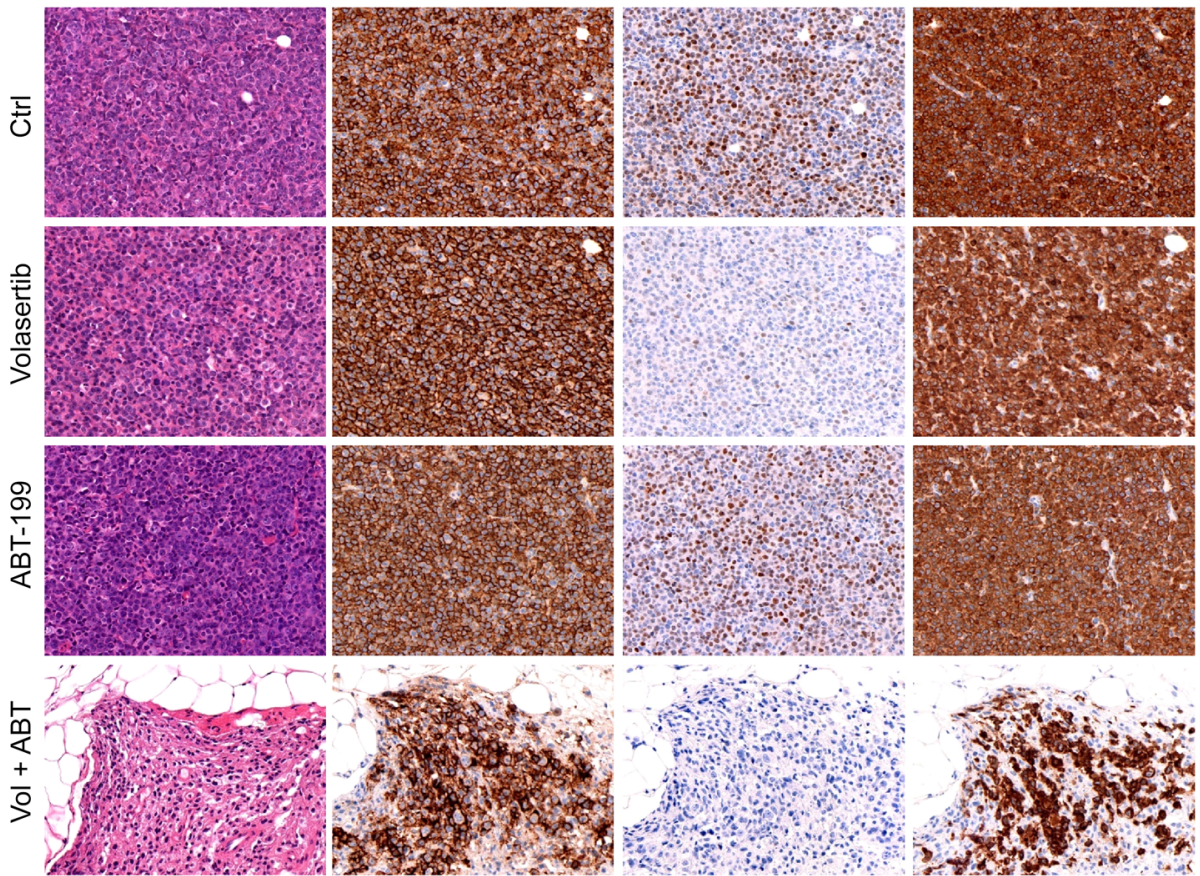

H
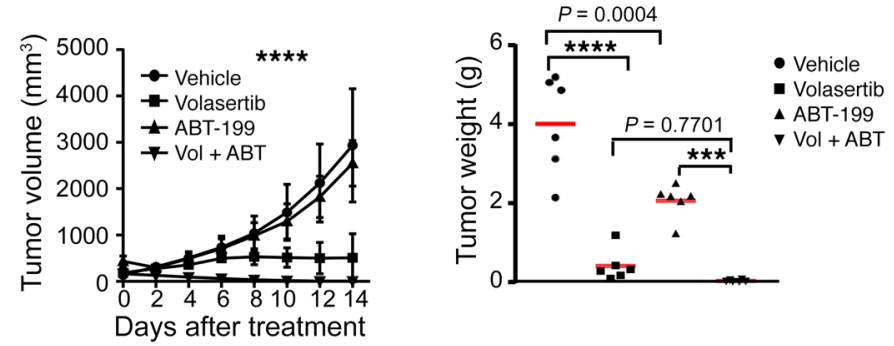
Figure 7. Dual PLK1/BCL-2 inhibition is a therapeutic strategy for DHL. (A) Combination treatment of volasertib (2 nM) with ABT-199 (DOHH2, U2932, $15 \mathrm{nM} ; \mathrm{CJ}, 40 \mathrm{nM}$ ) augments clonogenic suppression of DHL cells. (B) Combination treatment of volasertib plus ABT-199 (400 nM in VAL and PDX; 3.3 $\mathrm{MM}$ in patient [Pt] DHL specimens Pt-DHL1, Pt-DHL2, and Pt-DHL3) compromises survival of DHL cell lines (VAL cells shown), DHL PDX, and primary DHL patient specimens $(n=3)$ on a platform that recapitulates the lymphoma microenvironment. (C) Combination treatment of volasertib with ABT-199 has superior anti-DHL activity in VAL xenograft tumors. Left, tumor volume; right, tumor weight. ${ }^{* *} P=0.0002 ;{ }^{* * *} P<0.0001$. (D) Representative images of the tumors from the therapeutic study shown in C. (E) Levels of MYC, $p$-CSK3 $\beta$, total GSK3 $\beta$, and cleaved PARP in tumors from the therapeutic study shown in C. (F) IHC analyses of MYC, CD20, and BCL-2 protein expression in DHL PDX, and effects of volasertib or/and ABT199 treatment on their expression in this DHL PDX. Original magnification, $\times 40$. (G, H) Combination treatment of volasertib with ABT-199 provokes regression of DHL PDX tumors. (G) Representative images of tumors from the 4 cohorts of treated NSG recipient mice bearing DHL PDX tumors. (H) Tumor volume (left) and tumor weight (right) in the 4 cohorts of mice bearing DHL PDX. ${ }^{* * *} P=0.0003 ;{ }^{* * *} P<0.0001$. Results are shown as mean \pm SD of 6 animals/group for $\mathbf{C}$ and $\mathbf{F}$. Data shown in $\mathbf{A}$ and $\mathbf{E}$ represent mean $\pm S D$ or are representative of at least 3 independent experiments, respectively. Comparisons among group means in $\mathbf{C}$ and $\mathbf{F}$ were performed by 1-way ANOVA, followed by Tukey's multiple-comparison test. See complete unedited blots in the supplemental material.

that PLK1 signaling also regulates MCL-1 protein stability (Supplemental Figure 3E) to promote DHL cell survival. Further, CRISPR/ cas9-directed deletion of $F B W 7$ attenuated volasertib-induced MCL-1 degradation (Supplemental Figure 2B).

To further investigate the roles of the PLK1-MYC circuit in DHL, we performed ABPP profiling of DOHH and VAL DHL cells with or without treatment with volasertib for 2 or 12 hours (Figure 5D) to identify likely direct and indirect PLK1 downstream effectors. Volasertib-sensitive kinases that were also activated by MYC (Figure 1E) were then identified (Figure 5D and Supplemental Figure 4A). KEGG pathway analysis revealed that multiple kinase pathways, including AKT, ERK, cell cycle, and replicative stress, were both volasertib sensitive and activated by MYC (Figure 5D and Supplemental Figure 4, A and B). Finally, to assess whether volasertib treatment affects the transcriptional functions of MYC, we compared the MYC-regulated kinome and MYC target gene expression profiles (GEPs) with volasertib-sensitive kinases that are shared in volastertib-treated DOHH2 and VAL cells. Gene set enrichment analysis (GSEA) showed profound suppressive effects of volasertib treatment on the expression of MYC target gene sets and on the activity of MYC upregulated kinases in VAL and DOHH2 DHL cells (Figure 5E and Supplemental Figure 4C). Thus, PLK1 inhibition interferes with MYC-dependent transcription and the MYC-dependent kinome.

$B H 3$ profiles demonstrate $B C L-2$, but not $M C L-1$ or $B C L-X L$, dependence of DHL. BCL-2 plays key roles as an antiapoptotic protein in multiple hematological malignancies. As BCL2 is dysregulated in DHL via chromosomal translocation or amplification, it is considered as a prototype B cell malignancy with dysregulated BCL-2. Venetoclax (ABT-199), a potent and selective small-molecule BCL-2 inhibitor, is clinically being vetted as an effective therapy for many B cell lymphomas (37) and is postulated as being an ideal therapeutic for DHL. To test this notion, we performed ABT-199 drugresponse assays in $45 \mathrm{~B}$ cell lymphoma cell lines, including DHL cell lines (Figure 6A), as well as $\mathrm{BH} 3$ profiling in $5 \mathrm{DHL}$ lines ( $\mathrm{MYC}^{\text {hi }}$, BCL-2 ${ }^{\text {hi }}$ ) and 3 BL lines (MYChi, BCL-2 ${ }^{\text {lo }}$ (Figure 6C) and correlated their drug sensitivity with $\mathrm{BH} 3$ profiling results along with levels of BCL-2 family proteins, as assessed by Western blot. These analyses revealed that ABT-199 sensitivity is associated with both BCL-2 and MCL-1 protein expression levels (Figure 6B). $\mathrm{BH} 3$ profiling is a functional approach that measures the response of mitochondria to perturbation by a panel of $\mathrm{BH} 3$ domain peptides and can thus predict the cellular response to agents that target these individual proteins (BCL-2, MCL-1, BCL-XL). In comparison with BL cells, DHL cell lines (U2932, VAL, DOHH2, RC, CJ) are more reliant on BCL2-dependent mitochondrial apoptotic priming; i.e., overall DHL cells are more sensitive to BIM and BAD BH3 peptides (Figure 6C). These results indicate a BCL-2 dependency for DHL and provide a strong rationale for ABT-199 clinical application in DHL.

To gain insights into possible modes of resistance to ABT-199 in DHL, we also generated ABT-199-resistant DHL lines (VAL AR) by culturing cells in the presence of sublethal doses of ABT199 over 3 months. VAL_AR cells were then compared with parental VHL DHL cells using the $\mathrm{BH} 3$ peptide profiling assay. These analyses revealed that ABT-199-resistant VAL_AR cells were more dependent on MCL-1 compared with the parental cells (Figure 6D). Intriguingly, the VAL_AR ABT-199-resistant DHL cells also showed exquisite sensitivity to PLK1 inhibition (Figure 6E).

Combined PLK1/BCL-2 inhibition as a synthetically therapeutic strategy. The above data support the rationale for combining ABT199 with PLK1 inhibitors as a treatment regimen for DHL. Thus, we tested to determine whether combination therapies targeting BCL-2 and PLK1 have synergistic and durable anti-DHL activity ex vivo and in vivo. DHL cell lines were treated with ABT-199 or volasertib, singly and in combination, and were subjected to cell viability and clonogenic growth assays. The combination of volasertib with ABT-199 led to more profound inhibitory effects on DHL cell survival and clonogenic growth than cells treated with either volasertib or ABT-199 alone (Figure 7A). The potency of combination therapy was also assessed in primary DHL samples and PDX DHL samples using our ex vivo organotypic live-imaging cell-based platform in a reconstructed lymphoma microenvironment (24). In this system, primary DHL cells were seeded in a drug plate previously coated with human-derived stroma cells and collagen-1, and the drug screen was performed using volasertib or ABT-199 alone or in combination. Notably, the combination of volasertib with ABT-199 triggered more substantial reductions in DHL cell survival than those specimens treated with either volasertib or ABT-199 alone (Figure 7B).

To test potential efficacy in vivo, volasertib or ABT-199 was administered alone or in combination in NOD/SCID mice bearing DHL VAL tumors, after the xenograft had reached a volume of approximately $200 \mathrm{~mm}^{3}$. As predicted, volasertib induced substantial inhibition of DHL tumor growth, and the combination of volasertib with ABT-199 blocked DHL tumor progression (Figure 7, C and D). To assess the mechanism by which PLK1 inhibition abrogates the growth of DHL in vivo, we examined PLK1 target proteins using Western blots. As predicted, volasertib treatment in combination with ABT-199 markedly reduced levels of MYC and pGSK-3 $\beta$ and led to increases in cleaved PARP in these xenograft DHL tumor cells (Figure 7E). 
To further validate our in vivo and ex vivo findings, a previously described orthotopic (subrenal capsule) DHL PDX model in $N O D / S C I D / I L-2 R \gamma$ (NSG) recipient mice that is more reflective of human patients (38) was used to test responses to volasertib and volasertib in combination with ABT-199 treatment. For these studies, primary DHL cells isolated from a DHL patient were injected directly into the subrenal capsule of NSG mice. The PDXs established were then transferred into NSG mice, and the mice were randomly divided into 4 treatment cohorts. Histological examination and immunostains for CD20, MYC, and BCL-2 confirmed DHL diagnosis and also confirmed that the PDX lymphomas were comparable to the original diagnosed DHL (Figure 7F). NSG mice bearing the DHL PDX were treated (again when the tumors reached a volume of $\sim 200 \mathrm{~mm}^{3}$ ) with vehicle, ABT-199 alone, volasertib alone, or the combination of ABT-199 with volasertib. Tumor volume was assessed every other day up to 14 days after treatment. Consistent with prior observations of DHL, the tumors in all mice treated with ABT-199 alone showed partial growth suppression. In contrast, tumor volumes were markedly reduced with volasertib treatment, and tumors were completely eliminated in mice receiving the combination of ABT-199 and volasertib (Figure 7, G and H). No significant weight loss or movement disorders were observed after drug treatment (single or combined treatment). Notably, MYC expression was substantially diminished in volasertib-treated models (Figure 7E). Thus, PLK1 inhibition is an effective means to target MYC, and the combination of PLK1 and BCL-2 inhibitors is an attractive therapeutic approach for DHL.

\section{Discussion}

Dual dysregulation of the MYC and BCL-2 oncoproteins confers DHL cells with uncontrolled cell proliferation and resistance to apoptosis. As a consequence, DHL tumors rapidly progress and are drug resistant, and DHL patients have poor clinical outcome. For these tumors, as well as for other lymphomas bearing activated MYC, there is a dire need to develop new therapeutic strategies that disable the MYC oncoprotein. Notably, our findings, as well as those of others in neuroblastoma (26), reveal that MYC oncoproteins are no longer "undruggable," as targeting PLK1 kinase signaling provokes MYC destruction by the proteasome to induce a robust apoptotic therapeutic response. Excitingly, targeting PLK1 in combination with BCL-2 antagonists is revealed as a very attractive therapeutic combination that, at least in DHL PDX models, appears to eradicate disease.

Quantitative chemical proteomics and drug screens revealed unique kinome signaling and vulnerabilities in DHL. Further, BH3 peptide and BCL-2 family profiling demonstrated that select BCL-2 family dependencies can be identified in DHL and that some of these (e.g., MCL-1) are also dependent upon PLK1 signaling. Notably, in addition to PLK1, our studies suggest CHK1, Aurora A, and WEE1 as tractable therapeutic kinases that could show potency against DHL, particularly if inhibitors for these kinases are combined with the BCL-2 antagonist ABT-199. Indeed, these findings are in accord with those of others $(27,28)$ showing that targeting CHK1 and Aurora kinases is synthetically lethal for malignancies driven by MYC. Interestingly, all of these kinases control at some level replication stress, where signaling from these kinases restrains the extent of replication stress to allow proficient cellular proliferation, a hallmark of DHL. Finally, our profiling studies reveal potential mechanisms of resistance to ABT-199 in DHL, where MCL- 1 is revealed as a potential therapeutic for DHL patients that may develop resistance to ABT-199.

Heretofore the significance of PLK1 in the pathogenesis and management of this DHL was unknown. Our ABPP profiling, drug screens, and genetic and pharmacologic studies now establish PLK1 as a high priority target for DHL therapeutics, where: (a) $P L K 1$ is a direct or indirect MYC transcription target that is overexpressed in DHL; (b) PLK1 inhibitors such as volasertib show very potent activity against DHL cells ex vivo and in vivo, including those of primary DHL patients and DHL PDX; (c) PLK1 signaling is necessary to sustain MYC protein expression; and (d) PLK1 inhibitors in combination with the BCL-2 antagonist ABT-199 appear to eradicate tumors in validated models of DHL. In line with our findings, PLK1 is essential for mitosis and cell proliferation, where it promotes mitotic entry by phosphorylating cyclin B1 and CDK1 and initiates mitotic exit by activating the anaphase-promoting complex (APC) $(39,40)$. Further, forced overexpression of PLK1 promotes chromosome instability and aneuploidy by overriding the G2-M DNA damage and spindle checkpoints (41), and nonmitotic roles of PLK1 have also been suggested, including the regulation of cancer cell invasiveness and the protection against apoptosis (42). The latter effects of PLK1 are also consistent with our findings, where we have shown PLK1 signaling suppresses turnover of the antiapoptotic MCL-1 protein.

Collectively, our data reveal a positive, reciprocal activation circuit between PLK1 and MYC, which amplifies MYC-dependent oncogenic programs and promotes aggressive progression of DHL. First, PLK1 sustains MYC protein levels in DHL by enhancing MYC protein stability through activation of the AKT-GSK3 $\beta$ signaling axis. Several studies have shown that complex signaling pathways and several E3 ubiquitin ligases control MYC protein stability and that this is primed by the sequential phosphorylation of 2 highly conserved sites, S62 and T58 (21). These phosphorylation sites have opposing effects on MYC stability, where phosphorylation at S62 can stabilize c-MYC and then subsequent phosphorylation at T58 promotes MYC ubiquitin-dependent proteolysis. S62 phosphorylation can be directed by a number of kinases, including PLK1 itself, whereas T58 phosphorylation is mediated by GSK3 $\beta$ and leads to recruitment of the FBW7 E3 ubiquitin ligase (21). In neuroblastoma, it has been reported that PLK1 phosphorylates FBW7 and that this promotes FBW7 autoubiquitylation and destruction and stabilization of N-MYC (26). In contrast, in DHL, the administration of a potent and specific PLK1 inhibitor, volasertib, or PLK1 KO, leads to decreased levels of active p-AKT and p-GSK3 $\beta$, to a transient induction of $\mathrm{p}-\mathrm{T} 58-\mathrm{MYC}$ and then to marked reduction in MYC protein levels, and this is blocked by FBW7 depletion or the addition of proteasome inhibitors. Notably, unlike the scenario in neuroblastoma in which PLK1 inhibition leads to marked increases in the levels of FBW7 and concomitant reductions in N-MYC, in DHL, PLK1 appears to control FBW7 function without affecting its levels, as volasertib treatment does not affect FBW7 levels in DHL cells. However, similar to the findings for N-MYC in neuroblastoma (21), our ChIP results suggest that MYC also activates PLK1 transcription and that a positive feed-forward regulatory loop rein- 
forces MYC-regulated oncogenic programs in DHL. Collectively, these findings support the notion that a PLK1/FBW7/MYC signaling circuit underlies tumorigenesis and validate PLK1 inhibitors, alone or with BCL-2 antagonists, as potential effective therapeutics for MYC-overexpressing cancers.

As documented herein, PLK1 signaling is also required for stabilization of the antiapoptotic protein MCL-1 in DHL and this likely contributes the robust apoptotic response of DHL cells to volasertib treatment or PLK1 depletion. Quite strikingly, reductions in MCL-1 protein following PLK1 inhibition are also dependent upon FBW7, suggesting that similar PLK1-regulated signaling mechanisms control FBW7 activity to direct destruction of MYC and MCL-1 in DHL. These findings are also in accord with those showing FBW7-directed control of MCL-1 in other tumor types $(43,44)$.

Our findings strongly support the notion that PLK1 holds promise as a therapeutic target in DHL. In particular, there is a clear pharmacogenetic interaction between volasertib and MYC, in which volasertib treatment provokes destruction of MYC protein and for which the data suggest MYC status as a predictor of a robust therapeutic response to PLK1 inhibitors. Also compelling are the facts that (a) the expression of both MYC and PLK1 are markedly elevated in DHL; (b) there is a positive correlation between MYC and PLK1 mRNA and protein, and of MYC protein levels and active p-PLK1, in primary B cell lymphomas; and (c) elevated expression and activity of PLK1 connote poor outcome in DHL. Collectively, these observations implicate PLK1 as both a prognostic and predictive biomarker for B cell lymphoma patient diagnosis and treatment stratification.

Finally, using primary DHL patient specimens, xenograft studies with validated DHL cell line models, and DHL PDX, PLK1 inhibitors were revealed to show high potency versus this refractory malignancy. Thus, volasertib, a highly specific and potent PLK1 inhibitor $(45,46)$, has clinical potential for DHL patients, particularly as this agent has relatively minimal toxicity toward normal cells and hematopoietic progenitors (47). Further, our studies reveal that, when used in combination with the in-clinic BCL-2 antagonist ABT-199, PLK1 inhibitors provoke DHL tumor regression, a finding that is consistent with our $\mathrm{BH} 3$ peptide-profiling studies that demonstrate $\mathrm{BCL}-2$, but not MCL-1 or BCL-XL, dependence of DHL. ABT-199 has shown a high response rate as a single agent in refractory/relapsed CLL, and as a consequence, it has been granted breakthrough designation by the FDA for relapsed or refractory CLL (48). ABT199 has also been evaluated in DHL patients as a single agent, and although these patients showed initial high response rates to ABT-199, they eventually developed resistance, leading to accelerated mortality (49). Mechanistically, our BH3 peptideprofiling studies revealed that ABT-199 resistance in DHL cells is due to a switch to reliance on MCL-1, and consistent with this, we have recently shown that ABT-199 treatment triggers compensatory induction of MCL-1 (50). Notably, our data support the notion that PLK1 inhibition also confers MCL-1 dependence to DHL and that this is especially manifest in ABT-199resistant DHL. Collectively, these findings provide a proof of concept that supports clinical trials that will test the administration of a volasertib/ABT-199 combination therapeutic regimen for the treatment of DHL.

\section{Methods}

Patient samples and B cell lines. B cell lymphoma GEP data and DLBCL samples from the database of the University of Nebraska Medical Center were previously described $(51,52)$. The morphological and molecular diagnoses were performed by consensus pathology review. The survival data were updated annually and the OS (death from any cause) was estimated using the Kaplan-Meier method. Differences were assessed using the log-rank test. Differences among groups were considered significant at $P$ values below 0.05 .

All lymphoma cell lines were cultured in $10 \mathrm{mM}$ HEPES-buffered RPMI 1640 or Iscove's Modified Dulbecco's Medium (Gibco, Invitrogen) with penicillin $(100 \mathrm{U} / \mathrm{ml})$ and streptomycin $(100 \mu \mathrm{g} / \mathrm{ml})$ and maintained at $37^{\circ} \mathrm{C}$ in $5 \% \mathrm{CO}_{2}$. P493-6 B lymphoma cells were maintained with Tet system approved FBS (Takara Bio). The DHL lines CJ and RC (39) were a gift of Lan Pham. Other lymphoma lines used in this study were purchased from ATCC. Cell lines were routinely tested for mycoplasma using the Universal Mycoplasma Detection Kit from ATCC and were confirmed for identity using STR analyses by the Molecular Genomics Core of the Moffitt Cancer Center.

Antibodies and reagents. For Western blotting, the following antibodies were used: c-MYC (Abcam, catalog ab32072), $\beta$-actin (Santa Cruz Biotechnology Inc., catalog sc-47778HRP), PLK1 (Cell Signaling Technology, catalog 4513), p-PLK1 (Abcam, catalog ab155095), p-MYC-T58 (Abcam, catalog 185655), p-MYC-S62 (Abcam, catalog 185656), pan-AKT (Cell Signaling Technology, catalog 2920), p-AKT (ser473) (Cell Signaling Technology, catalog 4060), ERK1/2 (Cell Signaling Technology, catalog 9102), p-Erk1/2 (Thr202,Tyr204) (Cell Signaling Technology, catalog 9101), GSK-3 $\alpha / \beta$ (Cell Signaling Technology, catalog 5676), p-GSK-3 $\alpha / \beta$ (Ser21/Ser29) (Cell Signaling Technology, catalog 8566), MCL-1 (Santa Cruz Biotechnology Inc., catalog sc-12756), BCL-2 (Cell Signaling Technology, catalog 2872), BCL-xL (Cell Signaling Technology, catalog 2762), BAX (Cell Signaling Technology, catalog 2772), BAK (Cell Signaling Technology, cata$\log$ 12105), FZBW7 (Thermo Fisher Scientific, catalog 40-1500), and cleaved PARP (Cell Signaling Technology, catalog 5625).

Sources of reagents for cell culture studies and animal experiments were as follows: ABT-199 (Selleckchem), MK2206 (Selleckchem), PD0325901 (Tocris Bioscience), volasertib (Chemietek), MG132 (MilliporeSigma), SB216763 (Tocris Bioscience), and CHX (MilliporeSigma). Agents were dissolved in DMSO to a final concentration of $10 \mathrm{mM}$, aliquoted, and then stored at $-20^{\circ} \mathrm{C}$. Lithium chloride ( $\mathrm{LiCl}$ ) (MilliporeSigma), Tet (MilliporeSigma), and doxycycline (MilliporeSigma, D3447) were dissolved in water and stored at $-20^{\circ} \mathrm{C}$. Methocult medium was purchased from Stem Cell Technologies (H4034).

Gene overexpression and CRISPR/cas9 editing. Exogenous BCL-2 was overexpressed in lymphoma cells by retroviral transduction. Briefly, retroviral constructs were packaged by cotransfecting GP2HEK293T cells with p-CL-Ampho and p-MIP vector carrying EGFP or $B C L 2$. Stably expressing cells were established by puromycin selection. For CRISPR/cas9-mediated MYC KO, a gRNA targeting the MYC locus at the intron 1-exon 2 junction or gRNAs targeting PLK1 or FBW7 were cloned into a vector encoding espCas 9 and EGFP. The gRNA sequence for MYC is GCATCGTCGCGGGAGGCTGC. Lymphoma cells were transfected by the Neon Electroporation System (Life Technologies). GFP-positive cells were selected by flow cytometry and expanded as single clones. MYC KO was confirmed by Western blotting, and clones with deficient MYC expression were selected for further study. For 
the PLK1 or Fbw7 KD, CRISPR/cas9 gRNA constructs (Genescript) and the packaging vectors pVSVg (AddGene, 8454) and psPAX2 (AddGene, 12260) were used; transduction with a GFP CRISPR/ cas 9 gRNA expression construct served as a negative control. Forty-eight hours after transfection, culture supernatants containing virus were harvested and filtered. The filtrate was concentrated by ultracentrifuging for 2 hours at $100,000 \mathrm{~g}$ at $4^{\circ} \mathrm{C}$. Cells were infected with the concentrated viral supernatants and $8 \mu \mathrm{g} / \mathrm{ml}$ polybrene. On day 3 after infection, cell pellets were harvested, lysed, and analyzed by Western blotting. The gRNA target sequences for FBXW7 and PLK1 were as follows: FBXW7 CRISPR gRNA1: CTTACCCGTCTTCGACAAAA; FBXW7 CRISPR gRNA2: TGTATGTGTGTCCCGAGAAG; FBXW7 CRISPR gRNA3: CTCAGTATCAAACCGCTTCT; PLK1 CRISPR gRNA1: CTCCCCGTCATATTCGACTT; PLK1 CRISPR gRNA2: AGCCAAGCACAATTTGCCGT; and PLK1 CRISPR gRNA3: TACCTACGGCAAATTGTGCT.

ChIP assays. ChIP assays were performed using the SimpleChIP Enzymatic Chromatin IP Kit (Cell Signaling Technology). Briefly, $4 \times$ $10^{6}$ cells were first crosslinked by $1 \%$ formaldehyde for 10 minutes and the reaction was terminated with the addition of glycine. After a series of washes, nuclei were isolated and digested with $0.2 \mu \mathrm{l}$ micrococcal nuclease at $37^{\circ} \mathrm{C}$ for 20 minutes. The nuclear membrane was then disrupted by several pulses of sonication, and crosslinked chromatin was isolated. Chromatin digestion was confirmed by agarose gel electrophoresis and generated genomic DNA fragments with lengths of 200 to $500 \mathrm{bp}$. The digested chromatin was then incubated with anti-MYC antibody (Cell Signaling Technology, catalog 13987) or with normal rabbit IgG antibody (Cell Signaling Technology, catalog 2729), followed by pulldown with protein G magnetic beads. MYC-binding chromatin fragments were eluted by removal of magnetic beads and protein digestion with proteinase K. After DNA purification, binding to the PLK1 promoter region was assessed by qRT-PCR. MYC binding to the $C D K 4$ promoter-regulatory region was assessed as a MYCregulated positive control, as previously described (53).

$A B P P$. ABPP procedures were as detailed previously (24). Briefly, cell pellets were sonicated in IP/Lysis buffer (Pierce Kinase Enrichment Kit, Thermo Fisher Scientific), desalted, depleted of endogenous ATP with Zeba Spin Column (Thermo Fisher Scientific), and incubated with $10 \mu \mathrm{M}$ desthiobiotin-ATP probes at room temperature for 10 minutes. The labeled proteins were reduced, alkylated, and trypsin digested at $37^{\circ} \mathrm{C}$ for 2 hours. The labeled peptides were purified with high-capacity streptavidin agarose resin, washed, eluted, and subjected to LC-MS/ MS for peptide sequencing. ABPP experiments were performed as biological duplicates, and technical duplicates of each sample were analyzed by MS. Peptide identification and relative quantification were performed using MaxQuant software (version 1.2.2.5) (54).

High-throughput small-molecule drug screen (cell titer blue assays). Using a semi-automated platform, we tested an annotated library of 60 small molecules in DOHH2, VAL, U2932, SP53, CJ, and RC DHL cells. Cell viability was estimated by using Resazurin (R\&D Systems, catalog AR002). In brief, cells were seeded in 384-well plates with 2,000 cells per well in $30 \mu \mathrm{l}$ medium. Cells were cultured in the presence of the different compounds at serial 3-fold diluted concentrations. After 3 (kinase inhibitors) or 6 days (epigenetic inhibitors) of treatment, $6 \mu$ of Resazurin reagent was added into each well and incubated for 4 hours. Plates were read at a 560/590 nm wavelength to estimate cell proliferation.
Imaging- and cell-based drug screening assays. Cells were seeded in a 384-well plate of reconstructed bone marrow, including high physiological densities ( 1 to $10 \times 10^{6}$ cells $/ \mathrm{ml}$ ), extracellular matrix (collagen, fibronectin), and human bone marrow-derived stromal cells (BMSCs). A panel of drugs at 5 different concentrations was added to the media, the plate was continuously imaged for 96 hours, and a digital image analysis algorithm was used to detect cell viability based on membrane motion (pseudocolored in green). Changes in viability were quantified by AUC, as described previously $(24,55,56)$.

Xenograft studies. VAL-derived tumor xenograft studies were performed in NOD/SCID mice (The Jackson Laboratory). Briefly, 6-weekold female mice were subcutaneously inoculated on the flank with tumor cells $\left(8 \times 10^{6}\right.$ cells/animal $)$ suspended in $200 \mu \mathrm{l}$ PBS. Tumor volume $(V)$ was calculated based on the formula $V=L \times S^{2} \times 0.5$, where $L$ indicates the long axis and $S$ indicates the short axis. Volasertib was dissolved in DMSO $(30 \mathrm{mg} / \mathrm{ml})$ and diluted by $0.9 \% \mathrm{NaCl}$. ABT-199 was dissolved in DMSO $(50 \mathrm{mg} / \mathrm{ml})$ and then diluted at a final ratio of 5\% DMSO + 95\% $(0.9 \%$ solution) $\mathrm{NaCl}$. Drugs were given i.p., and the dose for both volasertib and ABT- 199 was $5 \mathrm{mg} / \mathrm{kg}$, once daily. When the xenograft reached a volume of approximately $200 \mathrm{~mm}^{3}$, mice were randomly assigned to the control, individual, or combined treatment groups, with 6 mice for each group. Animals were sacrificed when the control tumor reached approximately $2,000 \mathrm{~mm}^{3}$ or after the loss of more than $10 \%$ of body weight.

DHL PDX studies. The DEL PDX model (38) was a gift of Yuzhuo Wang (British Columbia Cancer Agency, Vancouver, British Columbia, Canada). PDX models were established in NOD/SCID and NSG mice (The Jackson Laboratory), respectively. Briefly, 6-week-old female mice were subcutaneously inoculated on the flank with tumor cells $\left(5 \times 10^{6}\right.$ cells/animal $)$ suspended in $100 \mu \mathrm{l}$ PBS. Tumor volume was calculated based on the formula $V=L \times S^{2} \times 0.5$, where $L$ indicates the long axis and $S$ indicates the short axis. Volasertib was dissolved in $0.1 \mathrm{~N} \mathrm{HCl}$ and diluted at $1: 10$ by $0.9 \% \mathrm{NaCl}$. ABT-199 was dissolved in DMSO and then diluted at a final ratio of $5 \%$ DMSO $+40 \%$ PEG 400 (or 300$)+5 \%$ Tween $80+50 \%$ (0.9\% solution) NaCl. Drugs were given i.p., and the dose for both volasertib and ABT-199 was 5 $\mathrm{mg} / \mathrm{kg}$. When the PDX xenograft reached a volume of approximately $200 \mathrm{~mm}^{3}$, mice were randomly assigned to the control, individual, or combined treatment groups, with 6 mice for each group. Animals were sacrificed when the control tumor reached approximately $2,000 \mathrm{~mm}^{3}$ or after the loss of more than $10 \%$ of body weight.

Statistics. One-tailed Student's $t$ test for homoscedastic variances was used to evaluate RT-PCR and apoptosis assay data. Statistical significance between the control and treated mice was evaluated using a 1-tailed Student's $t$ test. Group comparisons were performed using 1-way ANOVA with Tukey's post hoc test. $P$ values of less than 0.05 were considered significant. Data shown with the mean \pm SD are from at least 4 independent experiments. Statistical analysis was performed using GraphPad Prism, version 7.03.

Study approval. The human specimen study was approved by the Institutional Review Board at the University of South Florida (Tampa, Florida, USA), and patients provided signed informed consent forms. All animal studies were conducted in accordance with the NIH guidelines for animal care, and all animal procedures were approved by the IACUCs of the University of South Florida and Moffitt Cancer Center or by the IACUC of the University of Nebraska Medical Center. All experimental procedures and protocols were approved by the IACUC of the University of Nebraska Medical Center. 


\section{Author contributions}

JT, KF, YR, CB, JLC, and XZ designed experiments, interpreted data, and wrote the manuscript. YR, CB, XZ, T Lwin, T Li, and HJ performed functional, Western blot, molecular, ABPP, drug screen, colony formation, mouse model, data analysis, and research experiments. BF and JMK assisted with ABPP experiments. ASS, PRS, and KHS assisted with ex vivo drug screening assay and data analysis. BDS, JCC, and JV provided primary patient samples. CW and JY provided analysis of patient samples. LVP provided essential DHL lines. LW provided the gGFP plasmid, and XW performed statistical analysis. WSD, JV, LCM, KHS, JLC, and EMS provided essential reagents, intellectual support, and clinical samples. JMK, KHS, and JLC provided critical reading and editing of the manuscript.

\section{Acknowledgments}

We sincerely thank Yuzhuo Wang for providing the DEL PDX model (38). We are also grateful for expert technical support from members of the Tao, Fu, Cleveland, Koomen, Silva, and Shain laboratories for their input, and the Proteomics and Metabolomics, Molecular Genomics, Translational Research, Flow Cytometry, and Biostatistics and Bioinformatics Cores of the Moffitt Cancer Center for outstanding service. This work was supported in part by grants from the National Cancer Institute (NCI) (CA179062, CA134807, and CA137123 to JT and EMS), a grant from the Lymphoma Research Foundation (to KF), a sponsored research agreement from Incyte Corp. (to JT), the Nebraska Department of Health (LB506-18-22 to KF), NCI Cancer Center support grants (P30-CA036727 and P30-CA076292), the Cortner-Couch Chair for Cancer Research from the University of South Florida School of Medicine (to JLC), Lesa France Kennedy (to JLC), the Pentecost Personalized Research Foundation (to ASS and KHS), the H. Lee Moffitt Cancer Center Physical Sciences in Oncology (PSOC) grant (1U54CA193489-01A1), and funds from the state of Florida to the Moffitt Cancer Center and Research Institute. Access to primary DHL patient specimens was made possible through the Total Cancer Care Protocol at the Moffitt Cancer Center.

Address correspondence to: Jianguo Tao, Department of Laboratory Medicine and Hematopathology, 12902 Magnolia Drive, Tampa, Florida, 33612, USA Phone: 813.745.3885; Email: Jianguo. Tao@moffitt.org. Or to: Kai Fu, Department of Pathology and Microbiology, 42nd and Emile Avenue, Nebraska Medical Center, Omaha, Nebraska, 68198, USA. Phone: 402.559.7526; Email: kfu@unmc.edu.
1. Nilsson JA, Cleveland JL. Myc pathways provoking cell suicide and cancer. Oncogene. 2003;22(56):9007-9021.

2. Posternak V, Cole MD. Strategically targeting MYC in cancer. F1000Res. 2016;5:408.

3. Dave SS, et al. Molecular diagnosis of Burkitt's lymphoma. NEnglJMed. 2006;354(23):2431-2442.

4. Slack GW, Gascoyne RD. MYC and aggressive B-cell lymphomas. Adv Anat Pathol. 2011;18(3):219-228.

5. Aukema SM, et al. Double-hit B-cell lymphomas. Blood. 2011;117(8):2319-2331.

6. Marullo R, Rutherford SC, Leonard JP, Cerchietti L. Therapeutic implication of concomitant chromosomal aberrations in patients with aggressive B-cell lymphomas. Cell Cycle. 2016;15(17):2241-2247.

7. Sarkozy C, Traverse-Glehen A, Coiffier B. Double-hit and double-protein-expression lymphomas: aggressive and refractory lymphomas. Lancet Oncol. 2015;16(15):e555-e567.

8. Barrans S, et al. Rearrangement of MYC is associated with poor prognosis in patients with diffuse large B-cell lymphoma treated in the era of rituximab. J Clin Oncol. 2010;28(20):3360-3365.

9. Savage KJ, et al. MYC gene rearrangements are associated with a poor prognosis in diffuse large B-cell lymphoma patients treated with R-CHOP chemotherapy. Blood. 2009;114(17):3533-3537.

10. Li L, et al. Prognostic significances of overexpression MYC and/or BCL2 in R-CHOP-treated diffuse large B-cell lymphoma: A Systematic review and meta-analysis. Sci Rep. 2018;8(1):6267.

11. Shi QY, et al. MYC/BCL2 co-expression is a stronger prognostic factor compared with the cell-of-origin classification in primary CNS DLBCL. J Neuropathol Exp Neurol. 2017;76(11):942-948.

12. Green TM, et al. Immunohistochemical doublehit score is a strong predictor of outcome in patients with diffuse large B-cell lymphoma treated with rituximab plus cyclophosphamide, doxorubicin, vincristine, and prednisone. J Clin Oncol. 2012;30(28):3460-3467.

13. Johnson NA, et al. Concurrent expression of MYC and BCL2 in diffuse large B-cell lymphoma treated with rituximab plus cyclophosphamide, doxorubicin, vincristine, and prednisone. J Clin Oncol. 2012;30(28):3452-3459.

14. Hu S, et al. MYC/BCL2 protein coexpression contributes to the inferior survival of activated B-cell subtype of diffuse large B-cell lymphoma and demonstrates high-risk gene expression signatures: a report from The International DLBCL Rituximab-CHOP Consortium Program. Blood. 2013;121(20):4021-4031.

15. Perry AM, et al. MYC and BCL2 protein expression predicts survival in patients with diffuse large B-cell lymphoma treated with rituximab. $\mathrm{Br}$ J Haematol. 2014;165(3):382-391.

16. Farrell AS, Sears RC. MYC degradation. Cold Spring Harb Perspect Med. 2014;4(3):a014365.

17. Choi PS, et al. Lymphomas that recur after MYC suppression continue to exhibit oncogene addiction. Proc Natl Acad Sci U S A. 2011;108(42):17432-17437.

18. Dang CV, Reddy EP, Shokat KM, Soucek L. Drugging the 'undruggable' cancer targets. Nat Rev Cancer. 2017;17(8):502-508.

19. Llambi F, et al. A unified model of mammalian BCL-2 protein family interactions at the mitochondria. Mol Cell. 2011;44(4):517-531.

20. Montero J, Letai A. Why do BCL-2 inhibitors work and where should we use them in the clinic? Cell Death Differ. 2018;25(1):56-64.

21. Ruefli-Brasse A, Reed JC. Therapeutics targeting $\mathrm{Bcl}-2$ in hematological malignancies. Biochem J. 2017;474(21):3643-3657.

22. Manning G, Whyte DB, Martinez R, Hunter $\mathrm{T}$, Sudarsanam $\mathrm{S}$. The protein kinase com- plement of the human genome. Science. 2002;298(5600):1912-1934.

23. Knight ZA, Lin H, Shokat KM. Targeting the cancer kinome through polypharmacology. Nat Rev Cancer. 2010;10(2):130-137.

24. Zhao X, et al. Unification of de novo and acquired ibrutinib resistance in mantle cell lymphoma. Nat Commun. 2017;8:14920.

25. Pajic A, et al. Cell cycle activation by c-myc in a burkitt lymphoma model cell line. Int J Cancer. 2000;87(6):787-793.

26. Xiao D, et al. Polo-like kinase-1 regulates Myc stabilization and activates a feedforward circuit promoting tumor cell survival. Mol Cell. 2016;64(3):493-506.

27. Höglund A, et al. Therapeutic implications for the induced levels of Chk1 in Myc-expressing cancer cells. Clin Cancer Res. 2011;17(22):7067-7079.

28. den Hollander J, et al. Aurora kinases A and B are up-regulated by Myc and are essential for maintenance of the malignant state. Blood. 2010;116(9):1498-1505.

29. Tan J, et al. PDK1 signaling toward PLK1-MYC activation confers oncogenic transformation, tumor-initiating cell activation, and resistance to mTOR-targeted therapy. Cancer Discov. 2013;3(10):1156-1171.

30. Murga M, et al. Exploiting oncogene-induced replicative stress for the selective killing of Myc-driven tumors. Nat Struct Mol Biol. 2011;18(12):1331-1335.

31. Ferrao PT, Bukczynska EP, Johnstone RW, McArthur GA. Efficacy of CHK inhibitors as single agents in MYC-driven lymphoma cells. Oncogene. 2012;31(13):1661-1672.

32. Dauch D, et al. A MYC-aurora kinase A protein complex represents an actionable drug target in p53-altered liver cancer. Nat Med. 2016;22(7):744-753.

33. Sears R, Nuckolls F, Haura E, Taya Y, Tamai K, Nevins JR. Multiple Ras-dependent phosphor 
ylation pathways regulate Myc protein stability. Genes Dev. 2000;14(19):2501-2514.

34. Welcker M, Orian A, Grim JE, Grim JA, Eisenman $\mathrm{RN}$, Clurman BE. A nucleolar isoform of the Fbw7 ubiquitin ligase regulates c-Myc and cell size. Curr Biol. 2004;14(20):1852-1857.

35. Welcker M, et al. The Fbw7 tumor suppressor regulates glycogen synthase kinase 3 phosphorylation-dependent c-Myc protein degradation. Proc Natl Acad Sci U S A. 2004;101(24):9085-9090.

36. Yada $\mathrm{M}$, et al. Phosphorylation-dependent degradation of c-Myc is mediated by the F-box protein Fbw7. EMBO J. 2004;23(10):2116-2125.

37. Souers AJ, et al. ABT-199, a potent and selective BCL-2 inhibitor, achieves antitumor activity while sparing platelets. Nat Med. 2013;19(2):202-208.

38. Chapuy B, et al. Diffuse large B-cell lymphoma patient-derived xenograft models capture the molecular and biological heterogeneity of the disease. Blood. 2016;127(18):2203-2213.

39. Archambault V, Lépine G, Kachaner D. Understanding the Polo Kinase machine. Oncogene. 2015;34(37):4799-4807.

40. Zitouni S, Nabais C, Jana SC, Guerrero A, Bettencourt-Dias M. Polo-like kinases: structural variations lead to multiple functions. Nat Rev Mol Cell Biol. 2014;15(7):433-452.

41. Smits VA, Klompmaker R, Arnaud L, Rijksen G, Nigg EA, Medema RH. Polo-like kinase-1 is a target of the DNA damage checkpoint. Nat Cell Biol.
2000;2(9):672-676.

42. Rizki A, Mott JD, Bissell MJ. Polo-like kinase 1 is involved in invasion through extracellular matrix. Cancer Res. 2007;67(23):11106-11110.

43. Chen Y, et al. Bcl-2 family member Mcl-1 expression is reduced under hypoxia by the E3 ligase FBW7 contributing to BNIP3 induced cell death in glioma cells. Cancer Biol Ther. 2016;17(6):604-613.

44. Ye M, et al. Targeting FBW7 as a strategy to overcome resistance to targeted therapy in non-small cell lung cancer. Cancer Res. 2017;77(13):3527-3539.

45. Gjertsen BT, Schöffski P. Discovery and development of the Polo-like kinase inhibitor volasertib in cancer therapy. Leukemia. 2015;29(1):11-19.

46. Stadler WM, et al. An open-label, single-arm, phase 2 trial of the Polo-like kinase inhibitor volasertib (BI 6727) in patients with locally advanced or metastatic urothelial cancer. Cancer. 2014;120(7):976-982.

47. Nguyen T, et al. Synergistic interactions between PLK1 and HDAC inhibitors in non-Hodgkin's lymphoma cells occur in vitro and in vivo and proceed through multiple mechanisms. Oncotarget. 2017;8(19):31478-31493.

48. Roberts AW, et al. Targeting BCL2 with venetoclax in relapsed chronic lymphocytic leukemia. N EnglJ Med. 2016;374(4):311-322.

49. Davids MS, et al. Phase I first-in-human study of venetoclax in patients with relapsed or refractory non-Hodgkin lymphoma. J Clin Oncol.
2017;35(8):826-833.

50. Jiang $\mathrm{H}$, et al. Venetoclax as a single agent in combination with PI3K-MTOR1/2 kinase inhibitors against ibrutinib sensitive resistant mantle cell lymphoma [published online ahead of print January 30, 2018]. Br J Haematol. https://doi. org/10.1111/bjh.15079.

51. Huang X, et al. Activation of the STAT3 signaling pathway is associated with poor survival in diffuse large B-cell lymphoma treated with R-CHOP. JClin Oncol. 2013;31(36):4520-4528.

52. Li CT, et al. Cortical inhibitory and excitatory function in drug-naive generalized anxiety disorder. Brain Stimul. 2017;10(3):604-608.

53. Zhang $X$, et al. Coordinated silencing of MYC-mediated miR-29 by HDAC3 and EZH2 as a therapeutic target of histone modification in aggressive B-cell lymphomas. Cancer Cell. 2012;22(4):506-523.

54. Cox J, Mann M. MaxQuant enables high peptide identification rates, individualized p.p.b.-range mass accuracies and proteome-wide protein quantification. Nat Biotechnol. 2008;26(12):1367-1372.

55. Silva A, et al. An ex vivo platform for the prediction of clinical response in multiple myeloma. Cancer Res. 2017;77(12):3336-3351.

56. Silva A, Jacobson T, Meads M, Distler A, Shain K. An organotypic high throughput system for characterization of drug sensitivity of primary multiple myeloma cells. J Vis Exp. 2015;(101):53070. 